\title{
Transcriptome profile analysis of flowering molecular processes of early flowering trifoliate orange mutant and the wild-type [Poncirus trifoliata (L.) Raf.] by massively parallel signature sequencing
}

\author{
Jin-Zhi Zhang ${ }^{1}$, Xiao-Yan Ai ${ }^{1}$, Lei-Ming Sun ${ }^{1}$, Dong-Liang Zhang, Wen-Wu Guo ${ }^{1,2}$, Xiu-Xin Deng ${ }^{1,2}$, Chun-Gen Hu ${ }^{1 *}$
}

\begin{abstract}
Background: After several years in the juvenile phase, trees undergo flowering transition to become mature (florally competent) trees. This transition depends on the balanced expression of a complex network of genes that is regulated by both endogenous and environmental factors. However, relatively little is known about the molecular processes regulating flowering transition in woody plants compared with herbaceous plants.

Results: Comparative transcript profiling of spring shoots after self-pruning was performed on a spontaneously early flowering trifoliate orange mutant (precocious trifoliate orange, Poncirus trifoliata) with a short juvenile phase and the wild-type (WT) tree by using massively parallel signature sequencing (MPSS). A total of 16,564,500 and $16,235,952$ high quality reads were obtained for the WT and the mutant (MT), respectively. Interpretation of the MPSS signatures revealed that the total number of transcribed genes in the MT $(31,468)$ was larger than in the WT $(29,864)$, suggesting that newly initiated transcription occurs in the MT. Further comparison of the transcripts revealed that 2735 genes had more than twofold expression difference in the MT compared with the WT. In addition, we identified 110 citrus flowering-time genes homologous with known elements of flowering-time pathways through sequencing and bioinformatics analysis. These genes are highly conserved in citrus and other species, suggesting that the functions of the related proteins in controlling reproductive development may be conserved as well.
\end{abstract}

Conclusion: Our results provide a foundation for comparative gene expression studies between WT and precocious trifoliate orange. Additionally, a number of candidate genes required for the early flowering process of precocious trifoliate orange were identified. These results provide new insight into the molecular processes regulating flowering time in citrus.

\section{Background}

Flowering is one of the most important aspects of development in plants to ensure successful reproduction and eventual adaptation to surrounding environments. Plants have evolved mechanisms to integrate various environmental signals, including photoperiod and vernalization,

\footnotetext{
* Correspondence: chungen@mail.hzau.edu.cn

${ }^{1}$ Key Laboratory of Horticultural Plant Biology (Ministry of Education), College of Horticulture and Forestry Science, Huazhong Agricultural University,

Wuhan 430070, PR China

Full list of author information is available at the end of the article
}

to enable flowering under conditions that optimize seed production [1,2]. In recent years, molecular and genetic regulation of flower development has been extensively investigated in herbaceous plants, particularly in Arabidopsis [3-5]. A number of different pathways have been described in Arabidopsis that induce the floral transition, including vernalization, photoperiod, autonomous, and gibberellin (GA) pathways that form a regulatory network [6,7]. Genes involved in controlling the timing of floral transition have been identified through mutagenesis and analysis of natural variation. These four 
promotion pathways are integrated through the transcriptional regulation of two "flowering-time" genes, FLOWERING LOCUS T (FT) [8-10] and SUPPRESSOR OF OVEREXPRESSION OF CONSTANS 1 (SOC1), and two floral meristem identity genes, $L E A F Y(L F Y)$ and APETALA1 (AP1) [10-13]. Among the integrators, FT and SOC1 have a more direct function in determining flowering time. They share the common upstream regulators CONSTANS (CO), a key component in the long day pathway, and FLOWERING LOCUS C (FLC), a flowering repressor integrating autonomous and vernalization pathways [10]. FLC suppresses flowering, at least in part, by repressing the expression of the floral activators $S O C 1$ and $F T$, and it has been shown to bind directly to these activators $[14,15]$. $L F Y$ is a key player in the specification of floral meristem identity [16], and it dramatically increased the number of coflorescences in a lfy mutant due to the activity of AP1 [7]. Thus, crosstalk between pathways might explain how the multiple signals affecting flowering are coordinated, and differences in how pathways are integrated might underlie the diversity of plant flowering $[3,6,17]$.

Regulation of flowering in woody perennials shows remarkable differences with respect to herbaceous species. Annual plants complete their life cycle in one year and initiate flowering only once, whereas most other fruit crops have a long juvenile period, during which no reproductive development occurs. After this period, however, flowering happens repeatedly. How perennial woody plants undergo a long silent juvenile stage and then repeated vegetative growth and flowering has not been extensively studied at the level of transcription [17].

Citrus is one of the most widespread fruit crops in the world, with great economic and health value [18]. It is among the most difficult plants to improve through traditional breeding approaches due to undesirable reproductive traits and characteristics [19]. These include degrees of sexual sterility and incompatibility, nucellar embryony, extended juvenility, and large plant size. To accelerate flowering time, constitutive expression of $L F Y$ or AP1 derived from Arabidopsis was used to dramatically reduce generation time in citranges [20]. In a similar approach, but with a citrus gene, it was shown that transgenic poncirus carrying the CiFT (an FT homolog) also exhibited early flowering, although this phenotype was accompanied by several pleiotropic effects [21]. In addition, the long juvenile phase (6 to 8 years) of most citrus genotypes impedes the rapid evaluation of transgenic trees modified to affect adult traits such as fruit quality (e.g., rind and flesh color, flavors, maturity dates, seediness, abscission, peelability, rag content, acid and sugar levels) and other traits associated with productive mature trees. Thus, understanding the molecular regulation of the flowering process is crucial for controlling fruit production in citrus.

In 1976, precocious trifoliate orange with a short juvenile phase, a spontaneous mutant (MT) derived from Poncirus trifoliata (L.) Raf, was found in Yichang, Hubei province, China. In contrast to the 6- to 8-year juvenile period of the wild-type (WT) trifoliate orange, almost all of the seedlings that germinated from the MT had only a 3-year juvenile period; $20 \%$ of the seedlings even flowered in the year after germination. The MT seedlings can flower two to three times per year, while the WT plant flowers only once per year. The MT and the WT had nearly the same morphological characteristics aside from flowering habit, and no DNA polymorphisms were detected between them. Consequently, the MT was speculated to be a direct variant of the WT, which was ideal for studying floral induction, inflorescence development, and the flowering molecular mechanism. Therefore, a transcriptional study including a cDNA macroarray in combination with suppression subtraction hybridization $(\mathrm{SSH})$ was used to investigate gene expression changes in the MT, and a total of 368 differentially expressed genes were detected [22-25]. Interestingly, most of the 368 genes showed differential expression in the year after self-pruning, indicating that this period is a critical stage for the transcriptional regulation of the MT trait formation. Taken together, our previous research using SSH technology has provided important clues for understanding the formation of the mutation trait in precocious trifoliate orange; however, the transcriptional information from $\mathrm{SSH}$, especially for genes expressed at low levels, is rather limited. Further analyses of gene expression during the early flowering process is needed.

Massively parallel signature sequencing technology (MPSS), like expressed sequence tags (ESTs) and serial analysis of gene expression (SAGE), is a sequence-based method that can be used for transcriptional profiling to measure gene expression. However, MPSS provides more thorough qualitative and quantitative descriptions of gene expression due to its tremendous depth [26-30]. This depth enables identification of a nearly complete inventory of transcripts in a given sample [31]. To date, the MPSS method has provided a rapid way to identify and profile differentially expressed genes in a variety of plants, mutants, and tissues, and at different stages of development [28,30-32]. In this study, a genome-wide gene expression study was carried out between WT and precocious trifoliate orange by using MPSS, and a total of 36,523 genes were analyzed. Of these genes, 2735 showed a twofold or greater expression difference between the MT and WT. The results demonstrated that some genes may be newly transcribed in the MT. Our results also identified a large number of genes 
previously not known to be involved in the early flowering development process. Interpretation of the data solidified links between new information herein and our previous fragmentary knowledge, and provided new insight into the molecular processes regulating flowering time in citrus.

\section{Results}

Flowering characterization of WT and precocious trifoliate orange

Self-pruning is a physiologic phenomenon in trifoliate orange in which shoots cease vegetative growth by automatically withering the shoot tip $(0.5-1 \mathrm{~cm}$, Figure $1 \mathrm{~b}$ and 1e). Self-pruning is a necessary but insufficient condition for floral bud initiation. Until the late stage of self-pruning, the shoot apical meristem of juvenile trifoliate orange is in an undetermined state and floral primordia are not observed (October, Figure 1b). After self-pruning, the terminal bud and lateral buds of the juvenile trees enter dormancy until late February of the next year (Figure 1c). (In citrus, there are three flushes during the growing season. The spring flush is the most important one for growth and flower formation; these lateral buds develop into spring shoots in the next year.) A major characteristic of the MT is that its juvenile phase is shortened to 1 to 2 years, whereas the WT plant has a long juvenile period of 6 to 8 years. This "nonapparent" growth period is important for flower induction and the transition from the vegetative to the flowering stage of precocious trifoliate orange. Cytological observation revealed that the floral buds in the precocious mutant initiated differentiation immediately after self-pruning on spring shoots (Figure 1d-1f). Floral development hastened differentiation and produced the primordia of sepal, petal, stamen, and pistil sequentially. The whole flower bud integrates formation in one month, and then part of the flower bud population began to flower (Figure 1i-1m). However, most flower buds fell into dormancy until late February of the next year. For WT trifoliate orange, the spring shoots, which did not form floral buds, began to produce vegetative buds after self-pruning (Figure $1 \mathrm{~g}$ and $1 \mathrm{~h}$ ).

\section{MPSS signature abundance and distribution}

MPSS libraries were constructed using RNA extracted from the terminal bud and the five following buds of spring shoots (after self-pruning) for the MT and the WT. A total of 16,564,500 and 16,235,952 successful reads were produced for $\mathrm{MT}$ and $\mathrm{WT}$, respectively (Table 1). The sequence sets were filtered to remove low quality reads containing ambiguous nucleotides and adaptor sequences in both libraries, resulting in $16,067,565(97.00 \%)$ clean reads for MT and $15,701,789$ (96.71\%) clean reads for WT (clean reads are termed as "signature" hereafter). The signature sets were filtered to remove any signatures that were

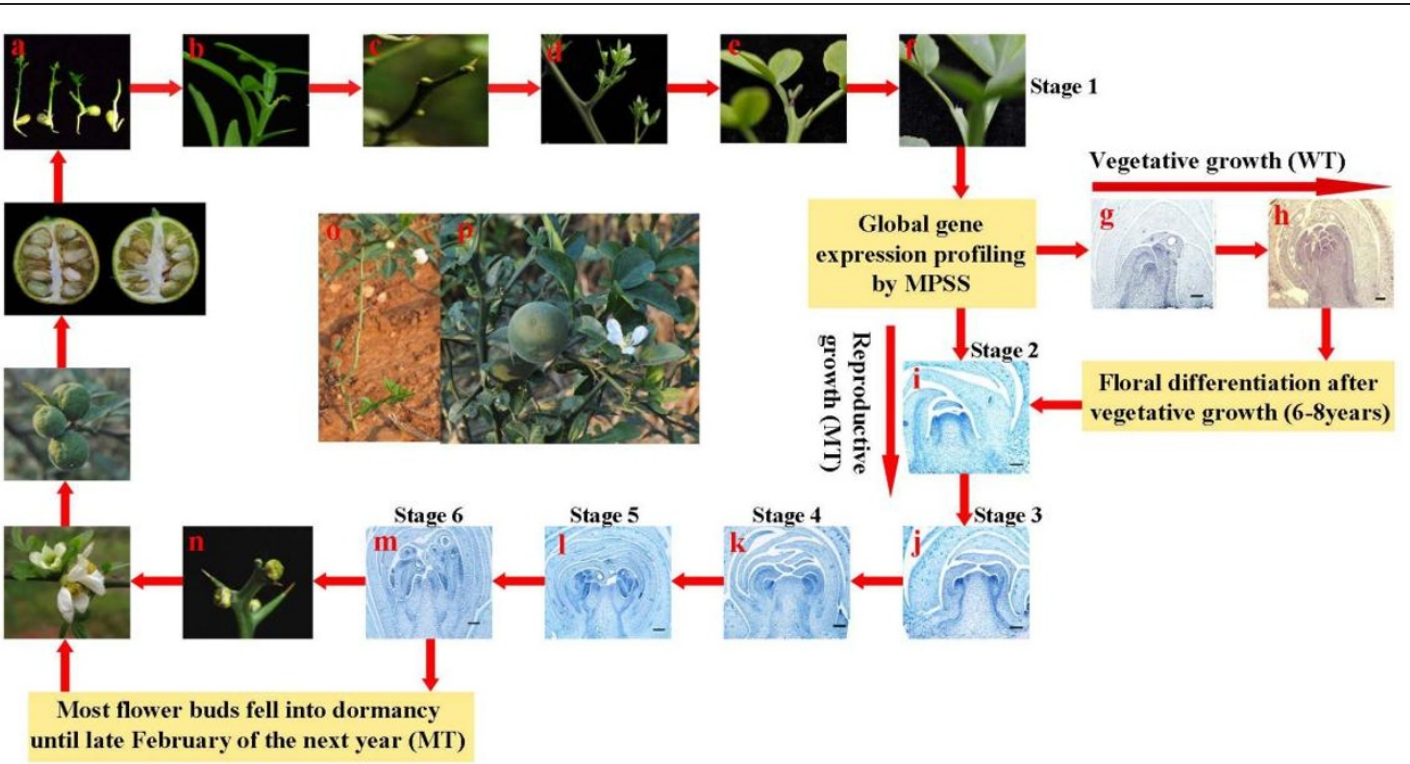

Figure 1 Schematic diagram of developmental stages involved in the flowering of precocious trifoliate orange. a, Seedling of precocious trifoliate orange ( 1 month). b. The shoot apical meristem of seedling begins self-pruning (October). c, The terminal bud and lateral buds of the juvenile trees became dormant after self-pruning (mid-October). $\mathbf{d}$, The lateral buds develop into a spring shoot in the next year (late February). e, The spring shoot begins self-pruning (early April). f, After self-pruning (mid-April). $\mathbf{g}, \mathbf{h}$, The lateral bud becomes a leaf bud (from late April to mid-May). i, Sepal primordia arise (late April). j, Petal primordia arise (late April). k, Stamen primordia arise (early May). i, Pistil primordia arise (mid-May). m, n, Full-developed floral bud (late May). o, p, The early flower morphology of precocious trifoliate orange. 
Table 1 Summary statistics of MPSS signatures in the precocious trifoliate orange (MT) and its wild-type (WT)

\begin{tabular}{lll}
\hline & MT library & WT library \\
\hline Total reads & $16,564,500$ & $16,235,952$ \\
Total nucleotides (nt) & $1,242,337,500$ & $1,217,696,400$ \\
High-quality reads & $16,067,565$ & $15,701,789$ \\
High-quality reads (\%) & 97.00 & 96.71 \\
Low quality reads & 496,935 & 534,163 \\
Low quality reads (\%) & 3 & 3.29 \\
GC percentage of high-quality reads (\%) & 44.64 & 45.06 \\
Reliable significant reads & $10,576,876$ & $10,527,303$ \\
Number of contigs & 83,412 & 81,148 \\
Number of singletons & 46,046 & 43,119 \\
Number of unigenes & 31,468 & 29,864 \\
\hline
\end{tabular}

not 1) reliable, i.e., observed in only one sequencing run, and 2) not significant, i.e., never observed at or above 3 transcripts per million (TPM) in either library. Of the $16,067,565$ signatures for MT, $34.2 \%$ $(5,490,689)$ did not meet the reliability criterion (Table 1 ). Meanwhile, of the $15,701,789$ signatures of the WT library, $33.0 \%(5,175,486)$ did not meet the reliability criterion. After filtering, 10,576,876 reliable signatures were observed in the MT library, with 10,527,303 reliable signatures in the WT library. The final set of reliable and significant signatures comprised 31,468 unigenes for the MT library and 29,864 unigenes for the WT library. When the data from the two libraries were combined, a total of 36,523 nonredundant unigenes were observed. Of these, 6,859 were not observed in the WT library and 5,055 were not present in the MT library (Table 1).

We found that MPSS was able to detect many transcripts expressed at low levels. Figure 2 shows the distribution of MPSS signatures at different abundance levels in TPM. The distribution of signature abundances across both libraries was generally quite similar. Three signatures in MT and four signatures in WT were expressed at a high abundance (more than $0.1 \%$, or $>$ 1000 TPM). While the number of signatures increased dramatically with a decrease in abundance, $74.88 \%$ of the total signatures in MT and $73.56 \%$ of the total signatures in WT had an abundance of less than $0.0001 \%$ (Figure 2). Moreover, of the signatures present in a given library, the vast majority, $93 \%$ of the WT library and $98 \%$ of the MT library, were below $0.001 \%$ abundance (< 100 TPM) (Figure 2). This illustrates the sensitivity of next generation sequencing technology in identifying transcripts with low expression. Of the total signatures, $1.6 \%$ of them were not found in the MT library and $1.7 \%$ of them were not found in the WT library (Figure 2).

\section{Differential expression of MPSS signatures between MT and WT plants}

The frequency of signatures was regarded as the relative expression level of each transcript in MT and WT libraries. Comparative analyses of the signature frequencies between MT and WT revealed that the expression ratio (MT/WT) varied greatly from 0.009 to 318 . Only $3.6 \%$ signatures were species specific, in that they were found only in one library and were absent from the other (expression ratio $=0$, Figure 3 ). Of the common signatures in both libraries, only $26.5 \%$ of all signatures (9670) showed a twofold or greater (ratio $>2$ or $<0.5$ ) expression difference between MT and WT, and were regarded as differentially expressed transcripts according to the criteria defined by Meyers et al. [33]. Of these, $19.9 \%$ had expression ratios between 2 and 5, and only a small percentage of signatures (3.6\%) showed more than a fivefold difference in expression level between the two libraries (Figure 3). Signature frequency was also compared statistically between the two libraries using the Zscore method according to Kal et al. [34], which uses the p-value and a statistical significance value. The expression of 2,735 signatures was significantly different at $\mathrm{p}<0.005$, at the same time their expression ratios were greater than 2 or less than 0.5 (Figure 4). Among these, 1000 genes were down-regulated in the MT compared to the WT and 1735 genes were up-regulated. Of these 2735 signatures, $1855(23 \%)$ were significant at $\mathrm{p}$ $<0.001$.

\section{Length distributions and functional annotation of unigenes}

The average length of unigenes was 674.41 and 665.35bp for the WT and MT library, respectively, which is shorter than those of Arabidopsis (1445bp) and soybean (1539bp) [35,36]. The median value for the length of the unigenes was 1020 and $1002 \mathrm{bp}$ for the WT and MT library, respectively, which is shorter than Arabidopsis (1459bp) and rice (1548bp) [37], but longer than poplar (990bp) [38]. The average length of the open reading frame was 718 and $711 \mathrm{bp}$, corresponding to an average polypeptide length of 239 and 237aa, respectively, which was also shorter than those of Arabidopsis, rice, and soybean, and longer than poplar and maize $[39,40]$. Additional File 1 provides the distributions of cDNA length and the CDS length from 31,468 and 29,864 nonredundant unigene sequences, respectively.

Our annotation method was based on sequence homology searches and the annotations that accompanied them. Its aim was to capture the most informative and complete annotation possible. Table 2 shows the hit numbers and percentages relative to those of the major public databases. These annotation statistics show all 


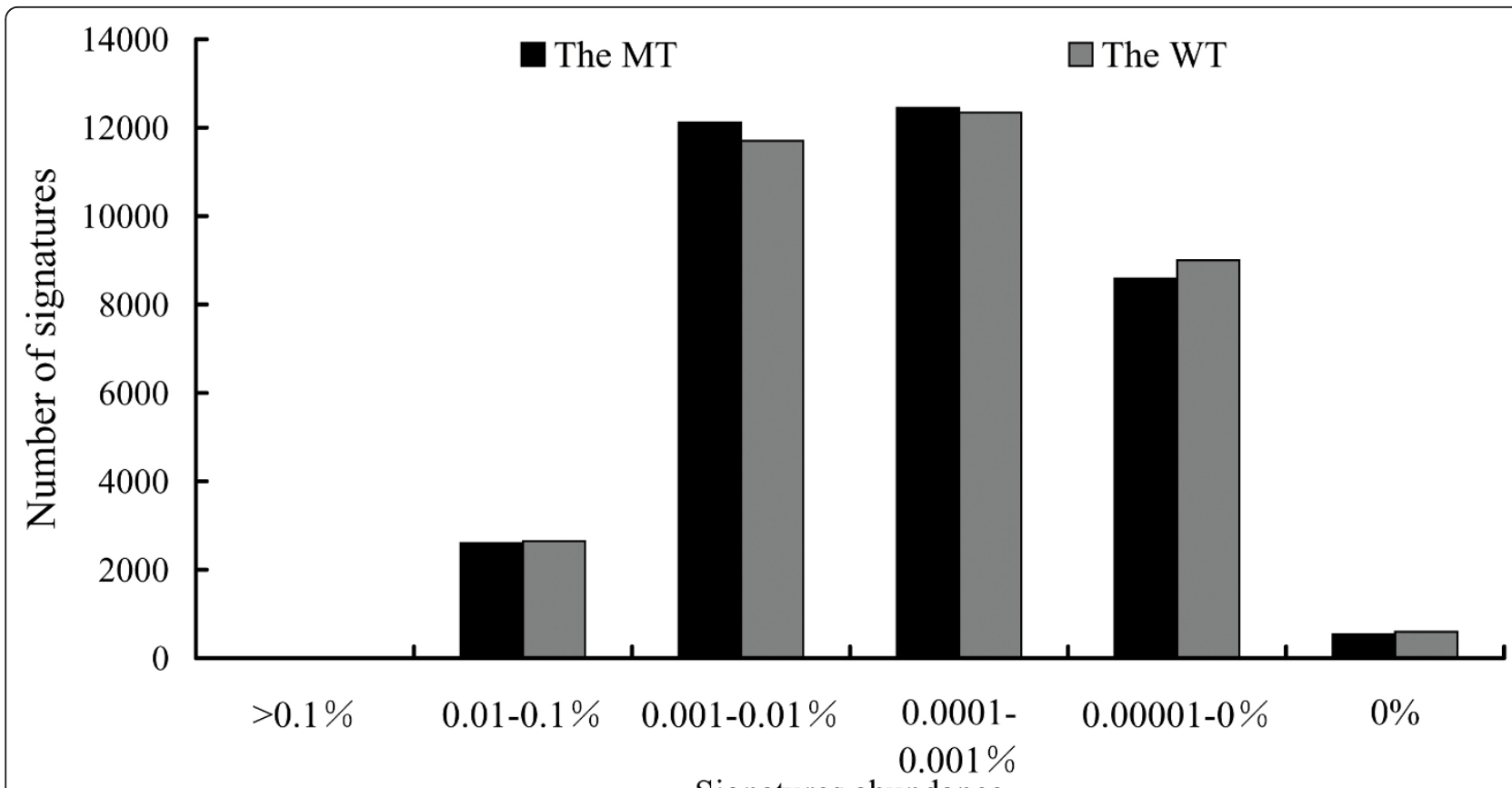

Signatures abundance

Figure 2 The MPSS signature abundance distributions. The abundance of each signature is calculated as a percentage of total signatures in the mutant (black column) and wild-type (gray column).

the unigenes annotated by the BLAST search against the public protein and nucleotide databases (Swiss Prot, KEGG, COG, and Nr) where the E-value threshold was set at 1E-5. Of the 31,468 MT and 29,864 WT unigenes, 23,835 and 23,076 unigenes had at least one hit within these databases, respectively (Table 2 ). The remaining unigenes $(24.26 \%$ and $22.73 \%$, respectively) that were not annotated likely comprised citrus-specific genes, as well as genes with homologs in other species whose corresponding biological functions have not yet been investigated. In addition, proteins with the highest ranks in the BLAST results were taken to decide the coding region sequences of the unigenes, and the coding region sequences were then translated into amino sequences with a standard codon table. Consequently, both the nucleotide sequences (5'-3') and amino sequences of the unigene coding region were acquired. Unigenes that cannot be aligned to any database were scanned by ESTScan [41] to get the nucleotide (5'-3') and amino sequences of the coding regions. A total of 25,318 genes $(12,064$ in sense and 12,254 in antisense) were identified in the MT library (Additional File 2), and 22,510 genes $(11,681$ in sense and 11,892 in antisense) were identified in the WT library (Additional File 3); a total of 7,150 (22.72\%) for the MT and 6,354 (21.28\%) for the WT library were not identified in sense or antisense. Transcription factors (TFs) are important regulators for activating or repressing the expression of coding or noncoding genes, through which they can further influence or control many biological processes [42]. Putative TF genes were identified by a BLAST search against rice, Arabidopsis, and Citrus sinensis TF genes downloaded from PlantTFDB (http://planttfdb.cbi.pku.edu. cn:9010/index.php) [43], identifying 569 putative TFs belonging to 60 TF families; there were 552 TFs in the WT library and 564. TFs in the MT library (Additional File 4). The $M A D S$ family was the most prevalent, followed by the AP2/EREBP and WRKY families. These results were slightly different from those of Arabidopsis [33] and rice [30], in which the $A P 2 / E R E B P$ family and zinc finger family were predominant, respectively. In addition, the $A P 2 / E R E B P$ family and $C 2 H 2$ family were slightly differentially expressed between the genotypes (Additional File 4).

\section{Functional classification of differential expression genes}

As a result of the completed genomic sequencing of the plant Arabidopsis, the currently available expressed sequences have been invaluable in defining the correct components of the gene structure in this species [44]. To evaluate the potential functions of genes with significant transcriptional changes between the MT and WT, Gene Ontology (GO) categories were assigned to the significant 2735 genes based on the TAIR GO slim provided by blast2GO. The categorization of differential expression genes according to the cellular component, 


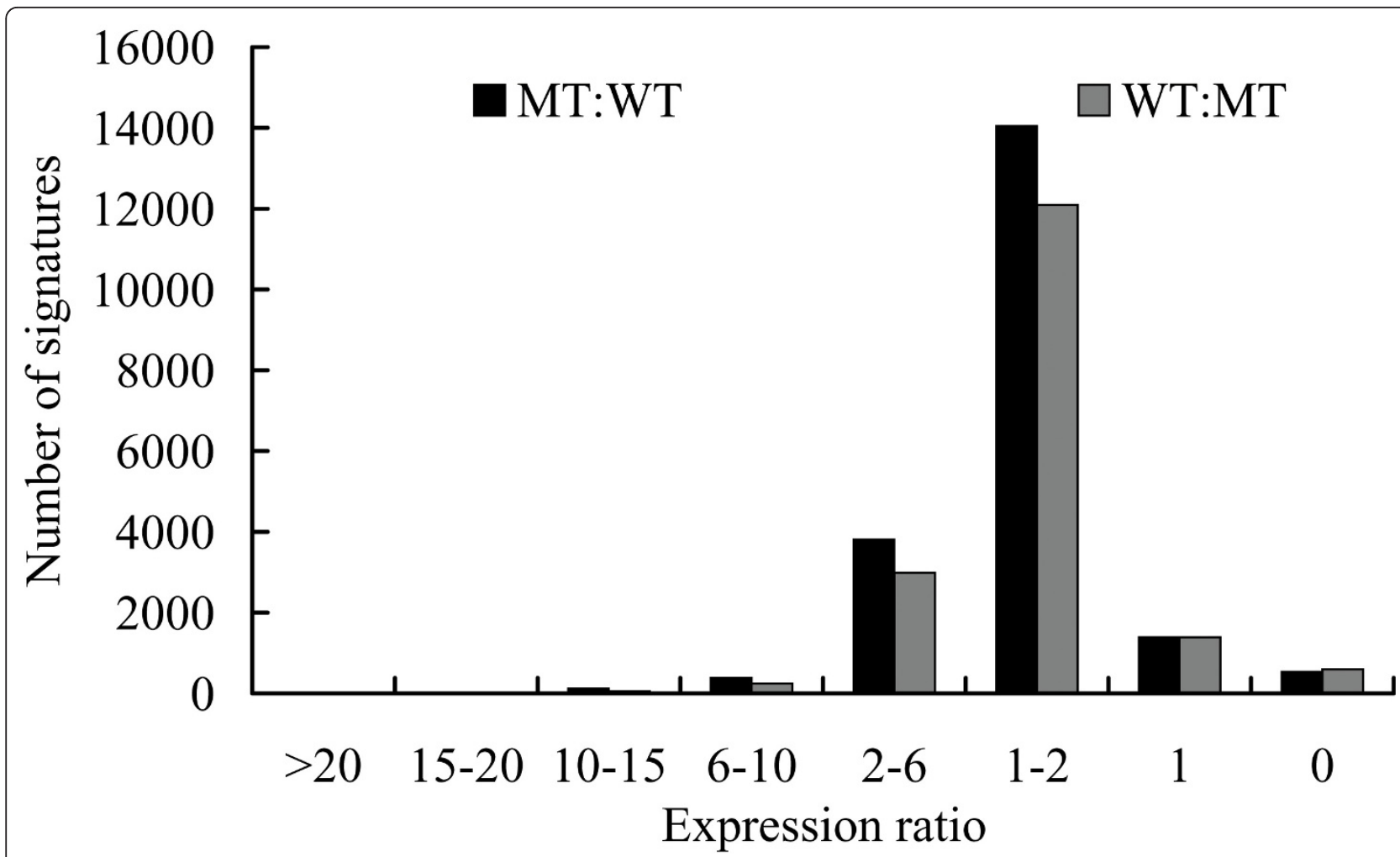

Figure 3 Comparison of expression of the MPSS signatures between the mutant (black column) and wild-type (gray column). The expression ratios compare the abundance of each signature between the mutant and wild-type. Columns denote the number of signatures with an expression ratio within the stated range.

molecular function, and biological process is shown in Figure 5. With regard to cellular component, the analysis revealed a high percentage of cell parts and organelles. For categories based on molecular function, the genes were finally classified into eight categories, as shown in Figure 5B; the three most overrepresented GO terms were binding (nucleotide binding, protein binding, chromatin binding), catalytic, and transcription regulators. Differential expression genes were related to 17 biological processes, including cellular process, biological regulation, metabolic process, developmental process, response to stimulus, multicellular organismal process, and others (Figure 5C). The biological interpretation of the significant differential expression genes was further completed using KEGG pathway analyses (Additional File 5). A total of 200 different metabolic pathways were found in this study, with some being consistent with biological processes already revealed by GO analyses. The most represented pathways included metabolic pathways (168 enzymes represented), biosynthesis of secondary metabolites (100), plant-pathogen interaction (65), phenylpropanoid biosynthesis (45), spliceosome (38), and cell cycle (26). Of these, some were related to mutation trait formation based on previous knowledge, including biosynthesis of plant hormones, spliceosome,
RNA degradation, ubiquitin-mediated proteolysis, and calcium signaling pathway. In addition, GO representations from this study were compared with a GO representation based on all the unigenes from sweet orange in the TIGR gene index database, and no significant differences were seen between the two groups.

\section{Identification of flowering-related genes}

There are physical, chemical, and biological signals that lead to the onset of flowering. The four known pathways that respond to these signals have been characterized in Arabidopsis and some herbaceous model plants. In order to identify flowering-related differential expression genes in this study, putative functions of 36,523 nonredundant signatures were assessed by BLAST searches against the TAIR9, RAP-DB, and NCBI Nr protein datasets. We identified 110 citrus unigenes representing putative homologs to flowering-related genes. Some of these genes are required for the day length response and some encode regulatory proteins specifically involved in the control of flowering, while others encode components of light signal transduction pathways or are involved in circadian clock function. A representation of the relationships among these processes is shown in Figure 6 and the putative homologs of the key players in 


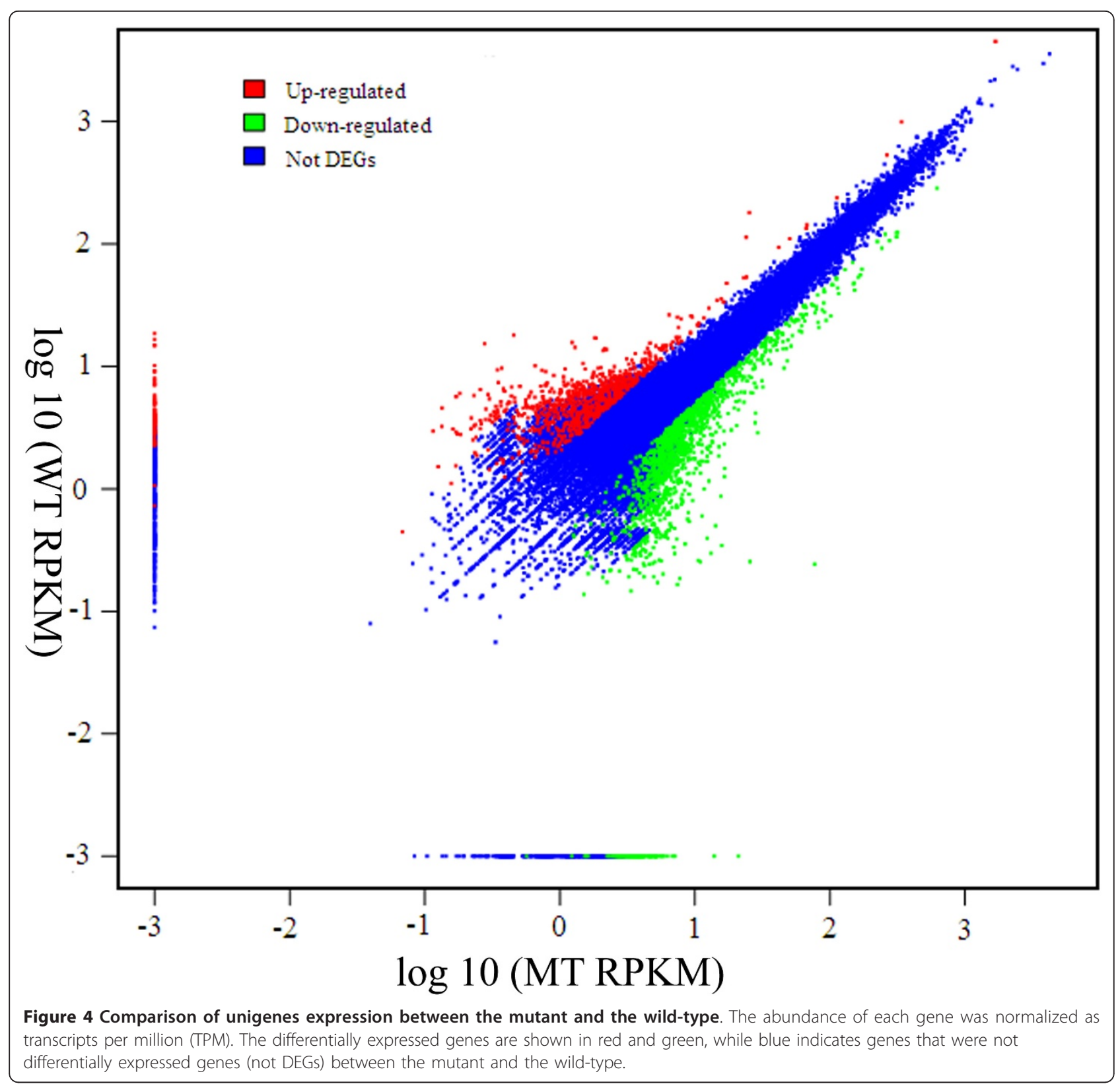

Table 2 Hit percentages against important public databases ${ }^{a}$

\begin{tabular}{lllllll}
\hline & $\mathbf{3 6 , 5 2 3}$ Unigenes (All) & \multicolumn{2}{l}{$\mathbf{2 9 , 8 6 4}$ Unigenes (WT) } & \multicolumn{3}{l}{$\mathbf{3 1 , 4 6 8}$ Unigenes (MT) } \\
\hline Database & Annotated $(\mathbf{n})$ & $\mathbf{\%}$ & Annotated $(\mathbf{n})$ & \% & Annotated (n) & \% \\
\hline SwissProt & 19,119 & 52.35 & 16,431 & 55.02 & 16,675 & 52.99 \\
KEGG & 12,724 & 34.84 & 11,005 & 36.85 & 11,279 & 35.84 \\
Nr & 26,938 & 73.76 & 22,042 & 73.81 & 23,770 & 75.54 \\
COG & 9,515 & 26.05 & 8,397 & 28.12 & 8,446 & 26.84 \\
Total & 26,993 & 73.91 & 23,076 & 77.27 & 23,835 & 75.74 \\
\hline
\end{tabular}

${ }^{\mathrm{a}} \mathrm{E}$ value threshold is $\mathrm{E}^{-5}$. 


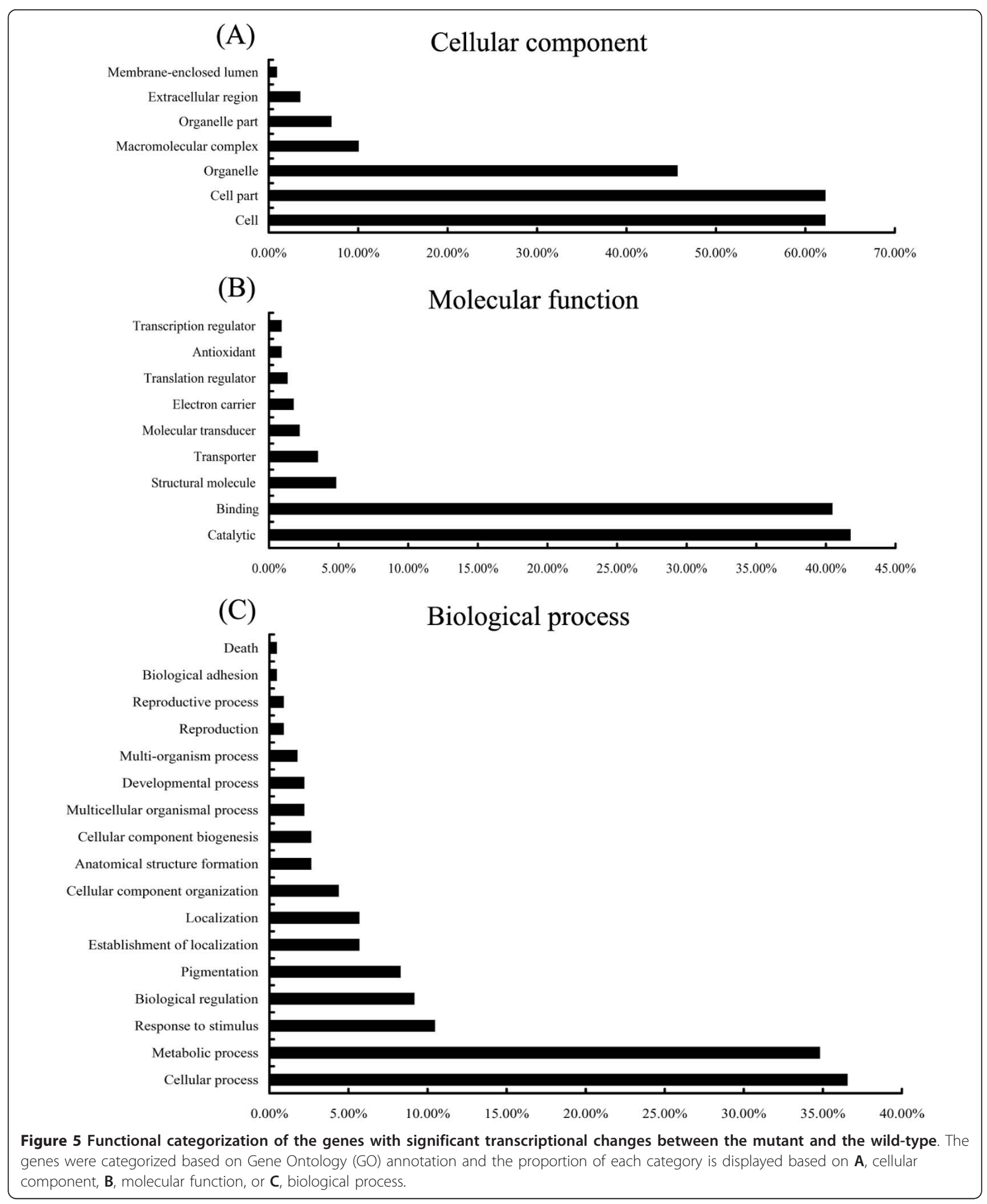




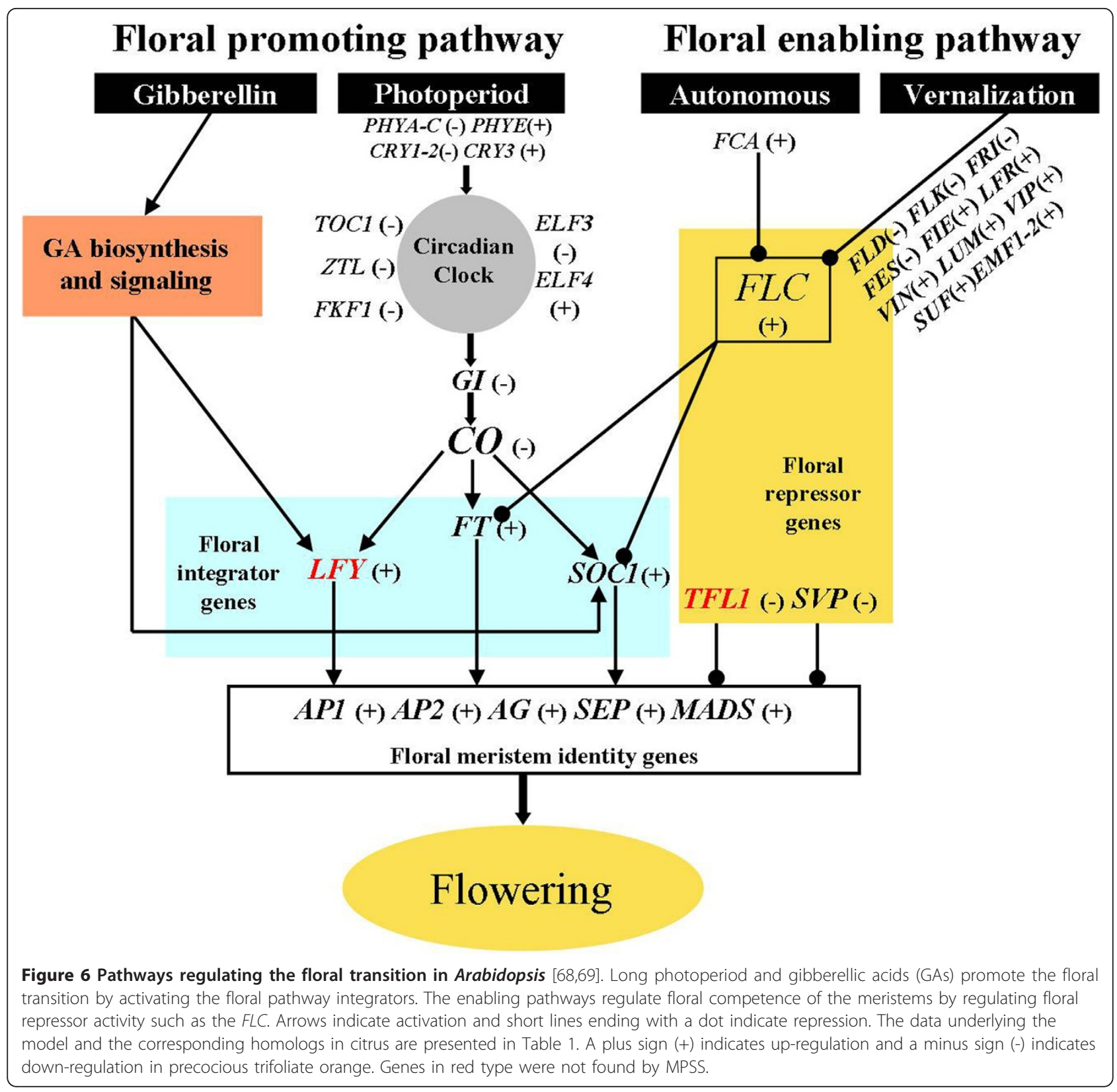

citrus are presented in Table 3. For example, we found some putative homologs for floral integrator or identity gene such as FT, SOC1, FLC, CO, and AP1.

Most genes of the Arabidopsis photoperiodic pathway were found in 36,523 nonredundant unigenes. We found several genes encoding putative photoreceptor apoproteins including Phytochrome A-C, Cryptochrome 1-3, ZEITLUPE (ZTL), and FLAVIN BINDING KELCH REPEAT F-BOX 1 (FKF1) (Table 3). In addition to genes of the photoperiodic pathway, homologs for both known sequences belonging to light quality pathways, PHYTOCHROME AND FLOWERING TIME 1 (PFT1) and RELATIVE OF EARLY FLOWERING 6 (REF6), were found. Of the central circadian clock genes, homologs of LATE ELONGATED HYPOCOTYL (LHY) and TIMING $O F C A B 1$ (TOC1) were also present in the two libraries (Table 3 ), but CCA1 was lacking. Furthermore, we found seven citrus unigenes that showed significant similarity to the Arabidopsis CO; these sequences were initially organized in six clusters. Among the regulators of $C O$ transcription and protein stability, GIGANTEA (GI) was identified in the two libraries (Table 3).

For the vernalization pathway, we were not able to find $M A F$-like sequences (MAF1-MAF5: $M A D S$ AFFECTING FLOWERING1-5) from our libraries by BLAST searches (Table 3 ). However, we identified 
Table 3 Citrus unigenes that share homology to flowering-time genes of other plants

\begin{tabular}{|c|c|c|c|c|c|c|c|}
\hline Gene ID & Gene Length & Reads (MT) & Reads (WT) & Accession number & Identity (\%) & E-value & Protein function (species) \\
\hline \multicolumn{8}{|c|}{ Photoperiod pathway } \\
\hline U8650 & 1327 & 379 & 348 & XM_002523031 & $728 / 1003(72 \%)$ & $5 \mathrm{E}-152$ & PHYA (Ricinus communis) \\
\hline U20394 & 1643 & 233 & 275 & XM_002512550 & $1175 / 1500$ (78\%) & 0 & PHYA (Ricinus communis) \\
\hline U1862 & 4042 & 2219 & 2668 & XM_002519184 & 2759/3356 (82\%) & 0 & PHYB (Ricinus communis) \\
\hline U11402 & 4270 & 748 & 829 & EU436651 & 1638/2059 (79\%) & 0 & PHYC (Vitis vinifera) \\
\hline U29320 & 871 & 16 & 24 & EU436656 & $541 / 690(78 \%)$ & $1 \mathrm{E}-163$ & PHYE (Vitis riparia) \\
\hline U2208 & 3284 & 710 & 700 & U39787 & 2162/2958 (73\%) & 0 & PHYE (Ipomoea nil) \\
\hline U8176 & 960 & 1809 & 1231 & NM_112521 & $329 / 460(71 \%)$ & $4 \mathrm{E}-57$ & PAP1 (Arabidopsis thaliana) \\
\hline U13637 & 1705 & 118 & 146 & XM_002275300 & 1396/1706 (81\%) & 0 & PIE1 (Vitis vinifera) \\
\hline U10186 & 1611 & 49 & 92 & XM_002275300 & 1052/1475 (71\%) & 0 & PIE1 (Vitis vinifera) \\
\hline U2658 & 3452 & 637 & 670 & XM_002275300 & 2232/2909 (76\%) & 0 & PIE1 (Vitis vinifera) \\
\hline U12327 & 1159 & 447 & 554 & NM_179665 & $59 / 75(78 \%)$ & $1 \mathrm{E}-06$ & PIL5 (Arabidopsis thaliana) \\
\hline U21857 & 3019 & 1115 & 1080 & NM_102365 & 1678/2388 (70\%) & 0 & PFT1 (Arabidopsis thaliana) \\
\hline U8820 & 1615 & 184 & 194 & NM_119618 & 220/271 (81\%) & $5 E-65$ & CIB1 (Arabidopsis thaliana) \\
\hline U11824 & 2226 & 89 & 148 & NM_120506 & $463 / 579(79 \%)$ & $1 \mathrm{E}-143$ & ELF6 (Arabidopsis thaliana) \\
\hline U9378 & 598 & 14 & 16 & NM_148863 & $212 / 268(79 \%)$ & $2 E-57$ & REF6 (Arabidopsis thaliana) \\
\hline U1219 & 3191 & 300 & 297 & NM_148863 & 287/371 (77\%) & $6 \mathrm{E}-74$ & REF6 (Arabidopsis thaliana) \\
\hline U9118 & 2795 & 3825 & 4339 & FJ882041 & 1486/1938 (76\%) & 0 & CRY2 (Fragaria vesca) \\
\hline U1555 & 3639 & 439 & 401 & NM_122394 & $359 / 463(77 \%)$ & $3 E-97$ & CRY3 (Arabidopsis thaliana) \\
\hline U9204 & 2765 & 1785 & 2109 & NM_116961 & 1364/1731 (78\%) & 0 & CRY1 (Arabidopsis thaliana) \\
\hline U12873 & 3154 & 940 & 911 & XM_002268467 & $2102 / 2575(81 \%)$ & 0 & FHY3 (Vitis vinifera) \\
\hline U20135 & 629 & 33 & 30 & AY830926 & $82 / 105$ (78\%) & $2 \mathrm{E}-27$ & ELF4 (Pisum sativum) \\
\hline U31613 & 682 & 7 & 20 & AY830926 & 153/213 (71\%) & $4 \mathrm{E}-24$ & ELF4 (Pisum sativum) \\
\hline U18048 & 2019 & 507 & 568 & NM_106379 & $352 / 491(71 \%)$ & $3 E-62$ & EFS (Arabidopsis thaliana) \\
\hline U9915 & 867 & 372 & 363 & EU916963 & 475/636 (74\%) & $4 \mathrm{E}-113$ & ELF4 (Citrus sinensis) \\
\hline U861 & 501 & 103 & 87 & EU916963 & $380 / 461(82 \%)$ & $5 \mathrm{E}-122$ & ELF4 (Citrus sinensis) \\
\hline U5852 & 687 & 497 & 341 & EU916963 & $661 / 669$ (98\%) & 0 & ELF4 (Citrus sinensis) \\
\hline \multicolumn{8}{|c|}{ Circadian clock } \\
\hline U10400 & 2627 & 907 & 1052 & DQ371901 & $1516 / 1849(81 \%)$ & 0 & ZTL2 (Glycine max) \\
\hline U17912 & 1648 & 1194 & 1240 & AY611028 & $803 / 1067(75 \%)$ & 0 & TOC1 (Castanea sativa) \\
\hline U12636 & 870 & 81 & 108 & AY611028 & $134 / 162(82 \%)$ & $1 \mathrm{E}-37$ & TOC1 (Castanea sativa) \\
\hline U10936 & 3030 & 753 & 1022 & NM_128153 & $94 / 109(86 \%)$ & $1 \mathrm{E}-25$ & ELF3 (Arabidopsis thaliana) \\
\hline U10110 & 3033 & 812 & 934 & AY371292 & $285 / 380(75 \%)$ & $7 E-60$ & ELF3 (Mesembryanthemum) \\
\hline U33021 & 293 & 12 & 17 & NM_102715 & $84 / 117$ (71\%) & $1 \mathrm{E}-06$ & RKF1 (Arabidopsis thaliana) \\
\hline U10089 & 381 & 17 & 14 & NM_102715 & $241 / 351(68 \%)$ & $4 \mathrm{E}-26$ & RKF1 (Arabidopsis thaliana) \\
\hline U12503 & 2363 & 229 & 258 & NM_130368 & 726/1031 (70\%) & $3 \mathrm{E}-127$ & RKF3 (Arabidopsis thaliana) \\
\hline U9215 & 3251 & 2563 & 2990 & XM_002524295 & 2328/2901 (80\%) & 0 & Gl (Ricinus communis) \\
\hline U7045 & 559 & 765 & 891 & XM_002524295 & 463/559 (82\%) & $5 \mathrm{E}-161$ & Gl (Ricinus communis) \\
\hline U9165 & 2436 & 716 & 860 & NM_105475 & 1303/1747 (74\%) & 0 & FKF1 (Arabidopsis thaliana) \\
\hline \multicolumn{8}{|c|}{ Vernalization pathway } \\
\hline U9585 & 1466 & 67 & 133 & NM_111874 & 1079/1457 (74\%) & 0 & FLD (Arabidopsis thaliana) \\
\hline U22618 & 1361 & 200 & 211 & NM_111874 & $367 / 527(69 \%)$ & $3 E-54$ & FLD (Arabidopsis thaliana) \\
\hline U23858 & 458 & 5 & 2 & NM_111874 & 141/185 (76\%) & $1 \mathrm{E}-28$ & FLD (Arabidopsis thaliana) \\
\hline U4972 & 283 & 72 & 95 & NM_111333 & 217/281 (77\%) & $8 \mathrm{E}-53$ & FLK (Arabidopsis thaliana) \\
\hline U2940 & 947 & 298 & 402 & NM_111333 & 143/171 (83\%) & $2 \mathrm{E}-42$ & FLK (Arabidopsis thaliana) \\
\hline U8380 & 1094 & 588 & 690 & NM_111333 & $531 / 714(74 \%)$ & $3 \mathrm{E}-122$ & FLK (Arabidopsis thaliana) \\
\hline U15692 & 502 & 860 & 282 & EU497679 & $327 / 327(100 \%)$ & $2 \mathrm{E}-165$ & FLC9 (Poncirus trifoliata) \\
\hline U12215 & 691 & 377 & 180 & EU605888 & 668/671 (99\%) & 0 & FLC7 (Poncirus trifoliata) \\
\hline U11512 & 2236 & 258 & 293 & XM_002511008 & 453/635 (71\%) & $2 \mathrm{E}-83$ & FRI (Ricinus communis) \\
\hline U2597 & 2060 & 637 & 638 & XM_002524905 & 1369/1728 (79\%) & 0 & FRI (Ricinus communis) \\
\hline U11002 & 1954 & 405 & 491 & XM_002529001 & 289/452 (63\%) & $8 \mathrm{E}-51$ & FRI (Ricinus communis) \\
\hline
\end{tabular}


Table 3 Citrus unigenes that share homology to flowering-time genes of other plants (Continued)

\begin{tabular}{|c|c|c|c|c|c|c|c|}
\hline U767 & 2311 & 257 & 227 & XM_002266277 & $289 / 452(63 \%)$ & $8 \mathrm{E}-51$ & FRI (Ricinus communis) \\
\hline U17929 & 1689 & 828 & 767 & NM_113199 & $926 / 1232(75 \%)$ & 0 & LFR (Arabidopsis thaliana) \\
\hline U14324 & 3620 & 950 & 928 & XM_002520611 & $621 / 839(74 \%)$ & $2 \mathrm{E}-144$ & LUM (Ricinus communis) \\
\hline U12966 & 1832 & 396 & 399 & EU884426 & $937 / 1258(74 \%)$ & 0 & VRN2-1 (Malus $\times$ domestica) \\
\hline U8773 & 1681 & 1784 & 1643 & EF064791 & $81 / 111(72 \%)$ & $9 \mathrm{E}-05$ & VIN3-1 (Arabidopsis thaliana) \\
\hline U17262 & 1894 & 191 & 161 & EF064791 & 218/294 (74\%) & $4 \mathrm{E}-41$ & VIN3-1 (Arabidopsis thaliana) \\
\hline U11331 & 1533 & 813 & 846 & EF064792 & 665/963 (69\%) & $1 \mathrm{E}-97$ & VIN3-2 (Arabidopsis thaliana) \\
\hline U17602 & 1306 & 951 & 885 & NM_119129 & $713 / 967$ (73\%) & $5 \mathrm{E}-171$ & VIP3 (Arabidopsis thaliana) \\
\hline U23612 & 1156 & 148 & 117 & NM_112785 & $38 / 47(80 \%)$ & 1.4 & VRN1 (Arabidopsis thaliana) \\
\hline U8880 & 1600 & 512 & 515 & NM_112785 & 284/384 (73\%) & $1 \mathrm{E}-59$ & VRN1 (Arabidopsis thaliana) \\
\hline U7754 & 1102 & 273 & 191 & NM_112785 & 127/183 (69\%) & $6 \mathrm{E}-11$ & VRN1 (Arabidopsis thaliana) \\
\hline U9616 & 1943 & 550 & 469 & NM_102836 & $748 / 1086$ (68\%) & $5 \mathrm{E}-110$ & SUF4 (Arabidopsis thaliana) \\
\hline U9937 & 953 & 38 & 41 & GQ177180 & $86 / 120(71 \%)$ & $1 \mathrm{E}-06$ & FES1 (Arabidopsis thaliana) \\
\hline U34190 & 1385 & 121 & 129 & NM_179890 & 176/259 (67\%) & $2 \mathrm{E}-13$ & FES1 (Arabidopsis thaliana) \\
\hline U12018 & 1939 & 288 & 248 & NM_121191 & $63 / 81(77 \%)$ & 7E-07 & EMF1 (Arabidopsis thaliana) \\
\hline U11876 & 2468 & 769 & 751 & XM_002281643 & $1519 / 2012(75 \%)$ & 0 & EMF2 (Vitis vinifera) \\
\hline$\underline{U} 2303$ & 1580 & 677 & 580 & NM_112965 & 784/1037 (75\%) & 0 & FIE (Arabidopsis thaliana) \\
\hline \multicolumn{8}{|c|}{ Autonomous pathway } \\
\hline U15708 & 416 & 21 & 23 & XM_002519206 & 146/185 (78\%) & $4 \mathrm{E}-33$ & FCA (Ricinus communis) \\
\hline U13760 & 1112 & 593 & 591 & XM_002519206 & 146/185 (78\%) & $4 \mathrm{E}-33$ & FCA (Ricinus communis) \\
\hline U9139 & 2236 & 883 & 868 & AK229352 & 75/97 (77\%) & $2 \mathrm{E}-10$ & FCA (Arabidopsis thaliana) \\
\hline U32661 & 983 & 8 & 8 & AK229352 & 75/97 (77\%) & $2 \mathrm{E}-10$ & FCA (Arabidopsis thaliana) \\
\hline U20071 & 955 & 126 & 82 & AK229352 & 75/97 (77\%) & $2 \mathrm{E}-10$ & FCA (Arabidopsis thaliana) \\
\hline \multicolumn{8}{|c|}{ Floral pathway integrator } \\
\hline U19004 & 346 & 6 & 3 & EU032531 & $167 / 170(98 \%)$ & $5 E-76$ & SOC1-1 (Citrus sinensis) \\
\hline U3347 & 353 & 22 & 8 & EU032532 & 343/353 (97\%) & $4 \mathrm{E}-166$ & sOC1- 2 (Citrus sinensis) \\
\hline U1570 & 283 & 8 & 6 & EU032532 & 244/281 (86\%) & $2 \mathrm{E}-98$ & sOC1-2 (Citrus sinensis) \\
\hline U27861 & 691 & 43 & 12 & AB301935 & 672/695 (96\%) & 0 & CiFT (Citrus unshiu) \\
\hline U27120 & 303 & 12 & 0 & AY338974 & 298/303 (98\%) & $1 \mathrm{E}-145$ & AP1 (Citrus sinensis) \\
\hline U17717 & 1389 & 1066 & 1165 & DQ371898 & $340 / 433(78 \%)$ & $5 E-96$ & CO2 (Glycine max) \\
\hline U2713 & 1871 & 799 & 962 & NM_001125127 & 279/375 (74\%) & $9 \mathrm{E}-56$ & CO9 (Arabidopsis thaliana) \\
\hline U17161 & 836 & 1317 & 1623 & FJ719767 & 538/716 (75\%) & $4 \mathrm{E}-132$ & CO (Mangifera indica) \\
\hline U8846 & 1586 & 2570 & 1905 & GQ864262 & $250 / 360(69 \%)$ & $2 \mathrm{E}-32$ & CO8 (Glycine max) \\
\hline U36503 & 1822 & 849 & 875 & GQ864265 & $114 / 140(81 \%)$ & $4 \mathrm{E}-28$ & CO (Glycine max) \\
\hline U9364 & 1051 & 128 & 123 & GQ864265 & $92 / 129(71 \%)$ & $4 \mathrm{E}-07$ & CO (Glycine max) \\
\hline U2300 & 1394 & 589 & 840 & GQ864263 & 116/147 (78\%) & $6 \mathrm{E}-25$ & CO9 (Glycine max) \\
\hline \multicolumn{8}{|c|}{ Other flowering genes } \\
\hline U5505 & 430 & 23 & 13 & U78949 & $331 / 396(83 \%)$ & $2 \mathrm{E}-114$ & MADS3 (Malus domestica) \\
\hline U13903 & 870 & 147 & 137 & DQ500880 & $470 / 617(76 \%)$ & $4 \mathrm{E}-126$ & MADS3 (Populus tomentosa) \\
\hline U1579 & 1210 & 152 & 93 & XM_002301057 & $526 / 669(78 \%)$ & $2 \mathrm{E}-156$ & MADS9 (Populus tomentosa) \\
\hline U6983 & 545 & 908 & 706 & AB218613 & $543 / 545$ (99\%) & 0 & CitMADS6 (Citrus unshiu) \\
\hline U34186 & 773 & 14 & 35 & AB218614 & 440/551 (79\%) & $2 \mathrm{E}-136$ & CitMADS8 (Citrus unshiu) \\
\hline U24167 & 336 & 9 & 5 & AB218611 & 286/336 (85\%) & 0 & CitMADS3 (Citrus unshiu) \\
\hline U12163 & 996 & 276 & 85 & AB218612 & $677 / 686(98 \%)$ & 0 & CitMADS5 (Citrus unshiu) \\
\hline U217 & 490 & 22 & 31 & NM_001084836 & 78/96 (81\%) & $1 \mathrm{E}-15$ & AGL16 (Arabidopsis thaliana) \\
\hline U9295 & 1938 & 2427 & 2378 & NM_101733 & 308/390 (78\%) & $9 \mathrm{E}-88$ & AGL65 (Arabidopsis thaliana) \\
\hline U8625 & 2045 & 400 & 316 & NM_105623 & 343/487 (70\%) & $1 \mathrm{E}-55$ & AGL94 (Arabidopsis thaliana) \\
\hline U20726 & 1826 & 257 & 166 & XM_002326128 & 450/604 (74\%) & 7E-102 & AP2 (Populus trichocarpa) \\
\hline U12861 & 1107 & 443 & 679 & FJ809943 & $818 / 820(99 \%)$ & 0 & AP2 (Poncirus trifoliata) \\
\hline U23896 & 275 & 11 & 3 & AY256859 & 210/277 (75\%) & $3 E-45$ & AP1 (Vitis vinifera) \\
\hline U24321 & 344 & 14 & 5 & GU357461 & 218/335 (65\%) & $2 \mathrm{E}-11$ & SEP1 (Euptelea pleiosperma) \\
\hline U4356 & 1096 & 319 & 190 & AB218614 & $627 / 636(98 \%)$ & 0 & CitMADS8 (Citrus unshiu) \\
\hline
\end{tabular}


Table 3 Citrus unigenes that share homology to flowering-time genes of other plants (Continued)

\begin{tabular}{|c|c|c|c|c|c|c|c|}
\hline U3656 & 2411 & 1156 & 1400 & NM_180137 & $534 / 715(74 \%)$ & $5 E-130$ & SPL1 (Arabidopsis thaliana) \\
\hline U12668 & 2514 & 1897 & 1917 & AJ011628.1 & $437 / 609$ (71\%) & 0 & SPL1(Arabidopsis thaliana) \\
\hline U9906 & 4081 & 1684 & 1828 & NM_101951 & 1803/2671 (67\%) & 0 & SPL14 (Arabidopsis thaliana) \\
\hline U3220 & 3148 & 854 & 790 & NM_180519 & $1221 / 1790$ (68\%) & $2 \mathrm{E}-168$ & SPL7 (Arabidopsis thaliana) \\
\hline U11760 & 1435 & 168 & 143 & HM018601 & $335 / 469(71 \%)$ & $8 \mathrm{E}-62$ & SPL10 (Vitis vinifera) \\
\hline U17573 & 1158 & 162 & 161 & XM_002517836 & $319 / 400$ (79\%) & $5 E-95$ & SPL (Ricinus communis) \\
\hline U31888 & 713 & 50 & 41 & FJ502237 & 595/639 (93\%) & 0 & SPL9 (Poncirus trifoliata) \\
\hline U23754 & 732 & 15 & 11 & FJ502238 & $563 / 576(97 \%)$ & 0 & SPL13 (Poncirus trifoliata) \\
\hline U12575 & 1329 & 71 & 81 & FJ502238 & $1272 / 1326(95 \%)$ & 0 & SPL13 (Poncirus trifoliata) \\
\hline U8652 & 1325 & 1020 & 1130 & NM_180137 & $734 / 1115(65 \%)$ & $9 \mathrm{E}-86$ & SPL1 (Arabidopsis thaliana) \\
\hline U9909 & 2323 & 99 & 162 & NM_105584 & 176/240 (73\%) & $1 \mathrm{E}-30$ & SPL6 (Arabidopsis thaliana) \\
\hline U12495 & 1047 & 460 & 397 & FJ373211 & $428 / 435(98 \%)$ & 0 & SVP (Poncirus trifoliata) \\
\hline U10522 & 1710 & 454 & 450 & AB290727 & 719/1066 (67\%) & $8 \mathrm{E}-88$ & TFL2 (Malus $\times$ domestica) \\
\hline
\end{tabular}

several homolog genes belonging to the FRIGIDA (FRI) complex as well as other regulatory complexes (FRIGIDA-ESSENTIAL1, SUPPRESSOR OF FRIGIDA4, and $M I N I D E P E N D E N S)$ involved in regulating the expression of $F L C$. Also putative members of $F L C$ repressing the PRC complex were present in the two libraries. These included putative VERNALIZATION INSENSITIVE 3 (VIN3), FERTILIZATION INDEPENDENT ENDOSPERM (FIE), EMBRYONIC FLOWER 1 (EMF1), VERNALIZATION 1 (VRN1), and LIKE HETEROCHROMATIN PROTEIN 1 (LHP1). In addition, a putative homolog for FLOWERING LOCUS D (FLD) sequence was identified in this study (Table 3); FLD plays a key role in regulating the reproductive competence of the shoot and results in different developmental phase transitions in Arabidopsis. Lesions in FLD result in hyperacetylation of histones in $F L C$ chromatin, up-regulation of FLC expression, and extremely delayed flowering [45].

In addition to the photoperiod and the vernalization pathways, we searched candidate genes for autonomous and GA pathways. Several sequences corresponding to Arabidopsis genes from both pathways were identified, suggesting the presence of these pathways in citrus. Among these genes we found homologs for Arabidopsis $F V E$ and $S V P$, which have been shown to control flowering in a specific thermosensory pathway. Moreover, some additional flowering-time regulators that have not been placed in any specific pathway were identified, such as the MADS transcription factor family genes, SQUAMOSA PROMOTER BINDING PROTEIN family genes, AGAMOUS family genes, and SEPALLATA family genes (Table 3).

\section{Differential expression of flowering genes between the MT and WT plants}

We compared the expression of selected flowering-time genes corresponding to each flowering pathway in the MT library and the WT library in order to explore the role of different pathways. Almost all flowering-related genes were differentially expressed between the genotypes; only a few of the genes were specifically expressed (Table 3). Of these, $A P 1$ was not observed in the WT library, although a citrus $A P 1$ had previously been identified [46]. The AP1 specifies flower meristem identity and is also required for normal development of sepals and petals. In the MT library, most flowering-promoting genes had slightly upregulated expression compared with the WT library, such as FLD, PFT1, and SEP1. Floral integrator genes ( $F T$, $S O C 1$, and $F L C$ ) were clearly up-regulated in the MT library (Figure 6). On the other hand, most repressors of flowering genes were slightly down-regulated in the WT library compared with the MT library (Figure 6).

\section{Verification of the genes related to MT trait formation}

Transcriptional regulation revealed by MPSS data was confirmed in a biologically independent experiment using quantitative reverse transcription polymerase chain reaction (RT-PCR). A total of 30 genes were chosen to design gene-specific primers (Additional File 6); these genes included 26 significantly differentially expressed genes, two genes of no differential expression, and two genes encoding proteins previously reported to be associated with, or involved in, developmental processes in other species. The transcript abundance patterns of the MT and WT were compared with MPSS data. Results showed that for 28 of the 30 genes, realtime PCR revealed the same expression tendency as the MPSS data, despite some quantitative differences in expression level. Figure 7 showed differential expression levels for 28 genes ( 20 for induced, three for repressed, three for antisense, and two previously reported flowering-related genes) between MT and WT. For example, the floral integrator gene $F T$ was up-regulated 1.7 times in MT compared with WT as analyzed by real-time PCR, consistent with MPSS data showing that the gene's expression in the MT was threefold higher than in the 
WT. Furthermore, the expression profile of six genes, including some citrus putative homologs for floral integrator or identity genes (SOC1 and $A P 1$ ), and three other newly detected genes with significant transcriptional changes were analyzed at six stages during flowering development between the MT and WT (Figure 8). As expected, the expression of these genes was correlated with floral induction, inflorescence development, and flowering of precocious trifoliate orange. It is notable that the expression levels of induced genes were upregulated in the spring shoot apex samples of WT and MT genotypes before the floral initiation had occurred and repressed genes were down-regulated (Figure 8).

\section{Discussion}

We explored expression patterns at a specific stage of flowering development in an early flowering trifoliate orange mutant by MPSS. MPSS and ESTs can be used for quantitative measurements of gene expression when combined with genomic sequencing [28,29]. Both approaches describe similar patterns of transcript abundance, although there are some clear differences that are perhaps associated with the methods themselves. Compared with EST technologies, MPSS involves deep transcript sampling and sequencing, usually 1-2 million transcripts per library, of a cDNA library on microbeads [29]. In principle, the MPSS data should provide a more thorough and quantitative representation of the absolute transcript population in terms of representation and relative abundance than EST data $[28,30,47,48]$. When compared with microarray technology that requires previous knowledge of genes, the limitations to detecting unknown genes are not encountered in MPSS $[48,49]$. On the other hand, compared to microarrays, a highthroughput sequencing approach is technologically more complex, yet much simpler statistically and methodologically [50]. The technology has been shown to provide comprehensive coverage and a sensitive measure of gene expression [51,52]. MPSS profiling has been used in studies to address various biological questions, including a whole-genome transcript analysis in Arabidopsis [53] and in human [51], and transcript expression profiling of hybrid and inbred parents in oyster [54]. From the results of this study, it is apparent that MPSS analyses not only highlight some genes and biological processes already revealed by our previous macroarray data $[23,24]$, but also reveal a large number of genes that are possibly involved in the formation of the early flowering trait. The data consistency from multiple approaches ensures that the MPSS data produced in this study are reliable. In addition, the approximately $3.6 \times 10^{8}$ base pairs of data produced here represent a substantial sequence resource and will contribute to genomic data available for citrus.
To understand the transcriptome profile and to isolate flowering-related genes during flowering development in citrus, genome-wide gene expression profiling was compared between WT and precocious trifoliate orange by using MPSS. The results revealed 2735 differentially expressed genes that were induced or repressed more than twofold in the MT at the $0.05 \%$ significance level. We identified a large number of newly discovered, intriguing unigenes of transcription and post-transcription factors in these differentially expressed genes, indicating that these genes may be key regulators that control flowering development by activating or repressing numerous genes (Figure 7). Additionally, a number of putative homologs of genes for flowering time and floral organ identity were also found. To obtain additional insights into the functions of the differentially expressed genes, we examined the GO categorization for the most similar Arabidopsis homologs of each gene using functions within the TAIR website. Functional category analyses revealed that a number of important pathways may work collaboratively in shaping the early flowering trait in the MT. Genes encoding proteins categorized as including binding (nucleotide binding, protein binding, and chromatin binding), biological regulation, and developmental processes were enriched among those genes differentially expressed between the WT and the MT. We found a large proportion of "no homology" and similarity to unknown proteins sequences in the GenBank database. The underlying genetics of flower induction and floral organ formation may differ between woody perennials and herbaceous model species. Some of these genes may play important roles from the vegetative phase to floral development in MT. In addition, a high proportion of antisense transcripts, i.e., 12,254 of 25,318 genes in MT and 11,892 of 22,510 genes in WT, were observed in our study. These results suggested that the early flowering trait could be regulated on transcriptional and posttranscriptional levels. In contrast, the MPSS data provided much more information for the regulation of these activities on the transcriptional level. A notable result is that a considerable percentage of the genes, $9 \%$ of the total annotated genes, were involved in the regulation of biological processes or transcription (Figure 5). The induction of regulatory genes of transcription correlates well with the increased overall transcription in the MT. The total number of transcribed genes in the MT was 31,468, more than in the WT $(29,864)$, suggesting that newly initiated transcription occurs in the MT. Moreover, analyses of the genes with transcription changes greater than twofold showed that up-regulated genes constituted $63.4 \%$ of the total changed genes. This suggests that these genes are related to uncharacterized mechanisms in the perception of signals and the initiation of flowering development. 


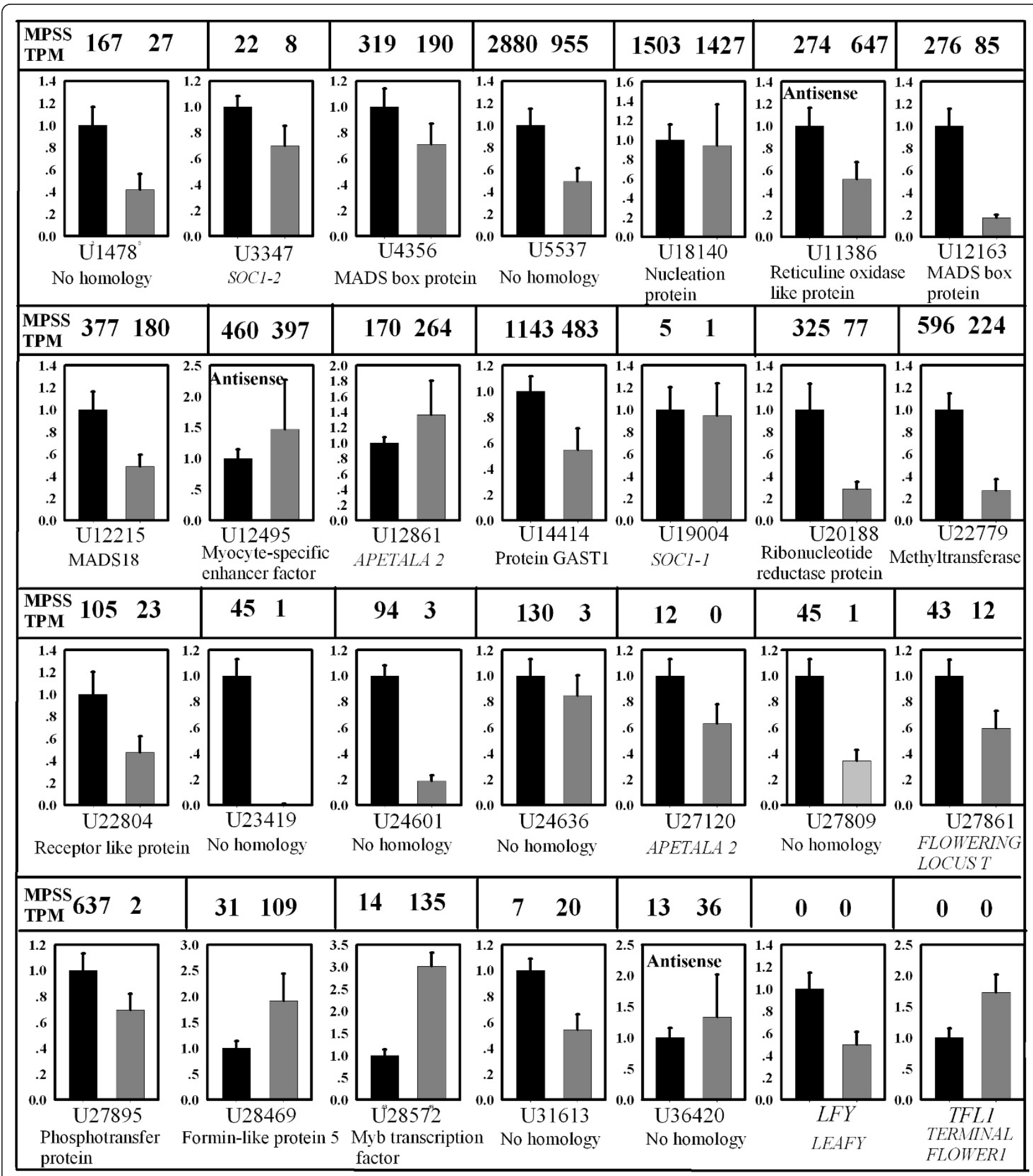

Figure 7 Real-time quantitative RT-PCR confirmation of the differentially expressed genes between the mutant (black columns) and the wild-type (gray columns). The transcript abundance from MPSS data is shown above each gene; TPM, transcripts per million. Relative transcript levels are calculated by real-time PCR with $\beta$-actin as the standard. Data are means \pm SE of three separate measurements.

For comprehensive identification of candidate genes in the citrus flowering pathways, we were able to identify 110 homolog genes among about 36,523 unigene sequences, representing putative citrus homologs to flowering-time genes (Table 3, Additional file 7). Sequences found in citrus corresponded to all known Arabidopsis flowering-time pathways, suggesting that all of these genetic pathways may be present in citrus. 

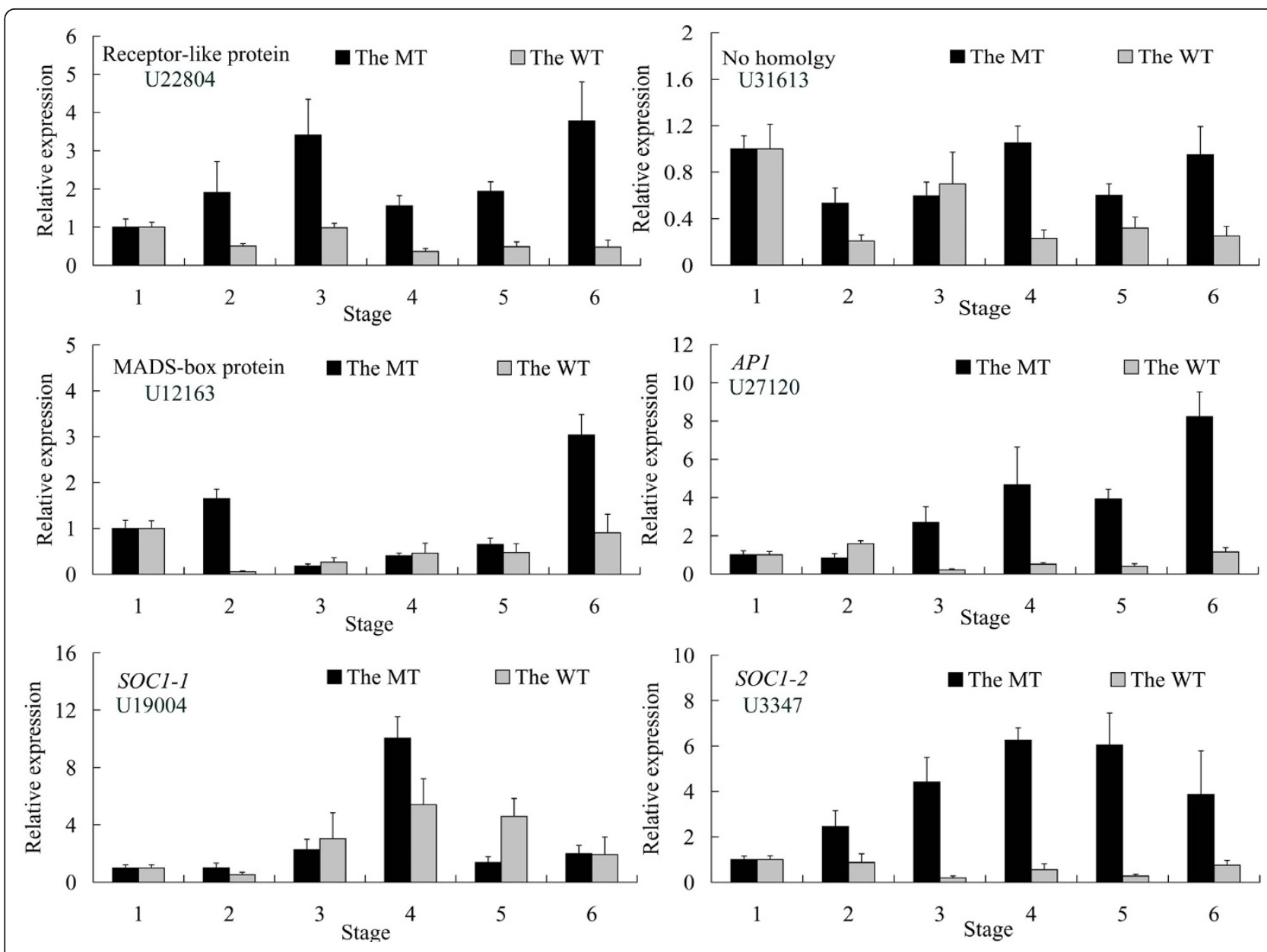

Figure 8 Transcript level of six selected genes at different stages of spring shoot development in the mutant (black columns) and wild-type (grey columns). Stage 1: After self-pruning (undetermined lateral bud); Stage 2: Sepal primordia arise; Stage 3: Petal primordia arise; Stage 4: Stamen primordia arise; Stage 5: Pistil primordia arise; Stage 6: Fully developed floral bud. Relative transcript levels are calculated by realtime PCR with Actin as a standard. Data are means \pm SE of three separate measurements.

Exogenous GAs have been shown to inhibit flower bud formation not only in citrus [55] but also in apples [56], pears [57], and cherries and peaches [58]. This hormone is thus believed to strongly participate in regulating flower bud formation. Many GA biosynthetic and catabolism-related genes were also found in this study; however, we did not find clear differences in the expression of GA biosynthetic and catabolism-related genes in the spring shoot apex samples of WT and MT genotypes before floral initiation occurred. So these data do not support the role of the GA pathway as the regulator of early flowering process in the MT, indicating that GA signal may be regulated during flowering development of the two gene types rather than only in the MT.

In Arabidopsis, the floral induction signals from these four major flowering pathways (photoperiod, autonomous, vernalization, and GA-induced pathways) are transmitted to three flowering pathway integrators, $F T$,
$S O C 1$, and $L F Y$. When $S O C 1$ is induced at the shoot apex, SOC1 together with AGL24 directly activates $L F Y$. $A P 1$, activated mainly by $F T$, is also necessary to establish and maintain flower meristem identity [59]. When $L F Y$ and $A P 1$ are established, flower development occurs at the anlagen of the shoot apical meristem according to the $\mathrm{ABC}$ model. During early flower development, $A P 1$ activates the A function and represses three redundantly functioning flowering time genes, SOC1, AGL24, and $S V P$ to prevent floral reversion. During late flower development, such repression is also necessary to activate SEPALATA3 (SEP3) which is a coactivator of $\mathrm{B}$ and C function genes with $L F Y$; otherwise SEP3 is suppressed by SOC1, AGL24, and SVP [59]. We have found citrus homologs for all of these genes except $L F Y$ and AGL24 in this study; one of the flowering-promoting genes was up-regulated in the MT library compared with the WT library (Table 3). Nevertheless, it is clear 
that the citrus genome contains orthologs to $L F Y$. Accordingly, overexpressing the citrus LFY DNA sequence dramatically induced early flowering in transgenic Arabidopsis [46]. Expression analysis of $L F Y$ by real-time RT-PCR showed that a transcript level of the gene was significantly up-regulated in the MT before the floral initiation had occurred (Figure 7). Therefore, we speculated that $L F Y$ is necessary to induce early flowering of precocious trifoliate orange. Citrus homologs for SOC1, FT, and AP1 were isolated previously and evaluated for their function in citrus or Arabidopsis $[21,46,60,61]$. They play important roles from the vegetative phase to floral development in citrus. $F T$ is a member of a small gene family in Arabidopsis that also contains TERMINAL FLOWER 1 (TFL1). Although TFL1 has been functionally characterized in citrus, we were not able to identify TFL1 sequences in this study. Our previous work also revealed that a large portion of the promotion of flowering by $F T$ and TFL1 was achieved through down-regulation of TFL1 levels and up-regulation of $F T$ levels in the MT [24]. One possible explanation for why these previously reported floweringrelated genes were not discovered in our library is that their mRNA transcript levels were too low to be measured by sequencing.

In Arabidopsis, $\mathrm{CO}$ encodes a zinc finger protein that acts as a floral activator and mediates the photoperiod pathway, whereas FLC encodes a MADS box protein that acts as a floral repressor and mediates the autonomous and vernalization pathways. $C O$ and $F L C$ regulate the expression of downstream genes, FT, SOC1, and LFY (Amasino 2010; Parcy 2005; Simpson and Dean 2002). We have identified seven unigenes showing significant (E-value lower than E-10) similarity to the Arabidopsis $\mathrm{CO}$ in two libraries. However, only a few of the CO-like genes were differentially expressed between the genotypes. Valverde et al. (2004) showed that the CO protein is ubiquitinated and then degraded by a protein complex called the proteasome, and that this process is regulated [62]. The autonomous and vernalization pathways promote flowering by repressing $F L C$ expression and many genes involved in the vernalization and autonomous pathways control the epigenetic status of the FLC chromatin [14]. Although FLC homologous MADS box genes have been recently found from several eudicot lineages by phylogenetic analysis [63], FLC homologous genes have not been identified in any woody plants except citrus. Previous work in this mutant showed that specific splice variants of $F L C$ were associated with transition from juvenile to mature trees, and four alternatively spliced transcripts of $F L C$ were isolated [22]. In this study, not only were alternatively spliced transcripts of putative FLC homolog found, but FRI homologs could also be found in the MT library and the WT library. FLC was up-regulated in the MT library compared with the WT library, and the FRI expression pattern was contrary to that of $F L C$. Our previous work provided evidence that the expression profile of $F L C$ was up-regulated during the winter, followed by a decrease in the spring and summer. This kind of cycling differs from the pattern observed in Arabidopsis [22]. A possible hypothesis suggests that due to the alternative splicing of PtFLC in citrus, which exerts its function in certain transcript form in the particular development stage, the total expression level of PtFLC was dispersed. Additionally, some sequences were found within the two libraries that would code for the other elements of the vernalization pathway: VRN1,VRN2, and VRN3 [64] or for the VIP3. VRN2 has a repressible role over the expression of $F L C$ and codes for a protein with homology to PcG proteins [65]. These results indicate that the vernalization pathway may be present in citrus. Meanwhile, these data also suggested that these genes may play a critical role in the early flowering process of precocious trifoliate orange.

\section{Conclusions}

In this study, we used the MPSS method to monitor global transcriptional changes in the MT compared with the WT, and identified 2735 differentially expressed genes that were induced or repressed more than twofold in the MT at $0.05 \%$ significance level. MPSS data analysis uncovered a large number of genes that were not previously known to be involved in formation of the mutation trait. A number of new genes possibly related to flowering time were found in this study. In addition, we explored 110 putative components for the genetic flowering pathways in citrus by identifying homologs of Arabidopsis flowering-time genes. The expression of selected flowering-time genes corresponding to each flowering pathway were compared in the MT library and the WT library, most flowering-promoting genes had up-regulated expression in the MT library, while most repressors of flowering genes had down-regulated expression in the MT library. These data also indicate that all known genetic flowering pathways may be present in citrus. The function of these elements can now be tested in heterologous systems, such as Arabidopsis, via transgenic approaches. We believe our results will be a valuable source for future research on the control of flowering and of biennial fruit-bearing patterns in citrus.

\section{Methods}

\section{Plant material and RNA preparation}

Wild-type and precocious trifoliate orange trifoliate orange samples were collected from the experimental fields of the National Citrus Breeding Center at Huazhong Agricultural University. The seeds of WT and 
precocious trifoliate orange trifoliate orange were planted in $20-\mathrm{cm}$ pots containing a potting mix of a commercial medium and perlite at a ratio of $3: 1$. The juvenile potted seedlings were then transplanted and grown under field conditions. These juvenile trees were watered regularly with a nutrient solution. The terminal bud and the five following buds (the major node position for flower formation) from spring flushes of these $\mathrm{MT}$ and WT trees were collected after self-pruning, sepal primordia arise, petal primordia arise, stamen primordia arise, pistil primordia arise, and full-developed floral bud, respectively. All materials were collected from three individual plants and immediately frozen in liquid nitrogen and stored at $-80^{\circ} \mathrm{C}$ until analyzed.

Total RNA was extracted according to a previous protocol [23]. A 1.2\% agarose gel, stained with ethidium bromide, was run to preliminarily indicate the integrity of the RNA. All RNA samples were quantified and examined for protein contamination $\left(\mathrm{A}_{260 / 280}\right)$ and reagent contamination $\left(\mathrm{A}_{260 / 230}\right)$ by a Nanodrop ND 1000 spectrophotometer. In addition, the RIN (RNA integrity number) determined by the Agilent Technologies 2100 Bioanalyzer was greater than 8.5 for all samples.

\section{Massively parallel signature sequencing}

The materials used for MPSS analyses were the terminal bud and the five following buds from MT and WT spring shoots after self-pruning, which occurs in mid-April (our previous results indicated that selfpruning is the critical stage for floral differentiation). Twenty micrograms of total RNA were sent to Beijing Genomics Institute (Shenzhen, China) where the libraries were produced and sequenced using Illumina's Genome Analyzer (Solexa). The MPSS was carried out essentially as in previous studies $[29,66]$, with modifications from Long SAGE [67]. Briefly, cDNA with polyA/T tail was prepared and digested with $D p n I I$ restriction enzyme. An adaptor with an $\mathrm{MmeI}$ recognition site was ligated to the 50 -end, followed by MmeI digestion that cut 21-22 bases downstream. This 21-22 base signature from each transcript was subsequently cloned by a unique adaptor and loaded to a microbead. This MPSS profiling process sampled 1-2 million sequenced transcripts per library on microbeads. Sequenced tags were generated by serial cutting and ligation of decoding adaptors. Sequencing runs were done by using two different cleavage steps, which are two different four-nucleotide sequencing frames offset by two bases (two-step) or three bases (three-step) [29]. The abundance of each signature was normalized to one million (transcripts per million, TPM) for the purpose of making comparisons between samples.

\section{MPSS data analyses}

To remove reads that might have arisen from errors in the MPSS procedure, two filters were applied to the derived reads [30]. The first, the "reliability filter," removed low quality reads containing ambiguous nucleotides or adaptor sequences. The second, the "significance filter," was intended to remove reads that were consistently present at background levels, excluding signatures lower than 3 TPM in both libraries according to the criteria described by Meyers et al. [30].

To link the expressed signatures to known genes from citrus, the unigene dataset from TIGR gene index database and Harvest database were combined to create a reference gene dataset. The signatures produced in silico were stored in the reference read database, through which the expressed MPSS signatures could be mapped on the corresponding EST contigs and singletons based on matches between MPSS signatures and in silico reads, as described previously [31]. The significance level of the differences of signature frequency and transcript abundance between the two libraries was analyzed using the Z-score method according to Kal et al. [34].

\section{Functional assignments of differentially expressed genes}

To assign putative functions to differentially expressed genes between MT and WT, annot8r program was run locally to BLAST against a reference database that stores UniProt entries, their associated Gene Ontology (GO), Enzyme Commission (EC), and Kyoto Encyclopaedia of Genes and Genomes (KEGG) annotation [56]. The GO categorization results were expressed as three independent hierarchies for biological process, cellular component, and molecular function [57]. The biological interpretation of the differentially expressed genes was further completed by assigning them to metabolic pathways using KEGG [58]. For the identification of pathways significantly affected by the mutation, we focused on the metabolite pathways with at least three affiliated genes.

\section{Real-time quantitative RT-PCR verification}

Thirty genes were chosen for confirmation by real-time quantitative RT-PCR with SYBR green I chemistry (QIAGEN, Germany). Primers for these genes were designed with the Primer Express software (PE Applied Biosystems, USA) and tested to ensure amplification of single discrete bands with no primer-dimers. Product size was about $180 \mathrm{bp}$. Total RNA (3 mg) was treated with $3 \mathrm{U}$ of DNase (Promega, USA) and then used in first-strand synthesis with an oligo (dT) primer (20-mer) and reverse transcriptase according to the manufacturer's instructions. For real-time PCR, an amount of cDNA corresponding to $25 \mathrm{ng}$ of input RNA was used in each reaction. Reactions were performed with the SYBR Green PCR Master Mix and analyzed in the ABI 
7500 Real-Time System. Real-time PCR products were amplified with $1 \mu \mathrm{l}$ of template of the RT reaction mixture, $10 \mu \mathrm{l}$ of $2 \times$ SYBR Green Master Mix, and $0.5 \mu \mathrm{l}$ of forward and reverse primer $(10 \mu \mathrm{mol} / \mu \mathrm{l})$, with water to a final volume of $20 \mu \mathrm{l}$. The levels of gene expression were analyzed with ABI 7500 Sequence Detection System Software (PE Applied Biosystems) and normalized with the results of $\beta$-actin. Real-time quantitative PCR was performed in four replicates for each sample, and data were indicated as means $\pm \operatorname{SD}(n=3)$.

\section{Additional material}

Additional file 1: Distribution of characteristic lengths of unigenes from the MT and the WT. Table 1: Length distribution of unigenes from the MT and the WT; Table 2: ORF length distribution of unigenes from the MT and the WT.

Additional file 2: Expression of unigenes in the WT. The table lists sense and antisense expression genes of the WT.

Additional file 3: Expression of unigenes in the MT. The table lists sense and antisense expression genes of MT.

Additional file 4: Transcription factors found in the WT and the MT. Putative TF genes were identified by a BLAST search against rice, Arabidopsis, and Citrus sinensis TF genes downloaded from PlantTFDB (http://planttfdb.cbi.pku.edu.cn:9010/index.php).

Additional file 5: The biological interpretation of the differential expression genes was further completed using KEGG pathway analysis. A total of 200 different metabolic pathways were found in this study, with some being consistent with biological processes already revealed by $\mathrm{GO}$ analyses.

Additional file 6: Primers used for real-time quantitative RT-PCR for the verification of MPSS data. Optimal oligonucleotide sequences for real-time RT-PCR were predicted by primer express program to prevent faint PCR products as primer dimmer and false amplicon

Additional file 7: The sequence information of citrus genes related to flowering development. Genes belonging to different flowering pathways are listed.

\section{Acknowledgements}

This research was supported financially by the National Natural Science Foundation of China (grant nos. 30971973, 30921002, 31071777), the Science Foundation of the Doctoral Discipline of the Chinese Ministry of Education (grant no. 20090146110009), the Fundamental Research Funds for the Central Universities (2010BQ044) and the 863 Project of China.

\section{Author details}

'Key Laboratory of Horticultural Plant Biology (Ministry of Education), College of Horticulture and Forestry Science, Huazhong Agricultural University, Wuhan 430070, PR China. ${ }^{2}$ National Key Laboratory of Crop Genetic Improvement, Huazhong Agricultural University, Wuhan, 430070, PR China.

\section{Authors' contributions}

ZJZ, AXY, HCG, and SLM are responsible for generating the MPSS data and for interpretation of the results. ZJZ and ZDL carried out the RT-PCR experiments. ZJZ drafted the manuscript. HCG and ZJZ participated in research design and statistical analyses. GWW, DXX, and HCG proposed and supervised the research. All authors read and approved the final manuscript.

Received: 11 August 2010 Accepted: 26 January 2011

Published: 26 January 2011
References

1. Li C, Dubcovsky J: Wheat FT protein regulates VRN1 transcription through interactions with FDL2. Plant J 2008, 55(4):543-554.

2. Kikuchi R, Handa H: Photoperiodic control of flowering in barley. Breed Sci 2009, 59(5):546-552.

3. Jack T: Molecular and genetic mechanisms of floral control. Plant Cell 2004, 16(suppl_1):S1-S17.

4. Koornneef M, Alonso-Blanco C, Peeters AJM, Soppe W: Genetic control of flowering time in Arabidopsis. Annu Rev Plant Biol 1998, 49(1):345-370.

5. Putterill J, Laurie R, Macknight R: It's time to flower: the genetic control of flowering time. Bioessays 2004, 26(4):363-373.

6. Boss PK, Bastow RM, Mylne JS, Dean C: Multiple pathways in the decision to flower: enabling, promoting, and resetting. Plant Cell Online 2004, 16(suppl_1):S18-S31.

7. Moon J, Lee H, Kim M, Lee I: Analysis of flowering pathway integrators in Arabidopsis. Plant Cell Physiol 2005, 46(2):292-299.

8. Kobayashi Y, Kaya H, Goto K, Iwabuchi M, Araki T: A pair of related genes with antagonistic roles in mediating flowering signals. Science 1999, 286(5446):1960-1962.

9. Kardailsky I, Shukla VK, Ahn JH, Dagenais N, Christensen SK, Nquyen JT, Chory J, Harrison MJ, Weigel D: Activation tagging of the floral inducer FT. Science 1999, 286(5446):1962-1965.

10. Samach A, Onouchi H, Gold SE, Ditta GS, Schwarz-Sommer Z, Yanofsky MF, Coupland G: Distinct roles of CONSTANS target genes in reproductive development of Arabidopsis. Science 2000, 288(5471):1613-1616.

11. Blázquez MA, Weigel D: Integration of floral inductive signals in Arabidopsis. Nature 2000, 404(6780):889-892.

12. Li D, Liu C, Shen L, Wu Y, Chen H, Robertson M, Helliwell CA, Ito T, Meyerowitz $\mathrm{E}, \mathrm{Yu} \mathrm{H}$ : A repressor complex governs the integration of flowering signals in Arabidopsis. Dev Cell 2008, 15(1):110-120.

13. Yoo SK, Chung KS, Kim J, Lee JH, Hong SM, Yoo SJ, Yoo SY, Lee JS, Ahn JH: Constans activates suppressor of overexpression of constans 1 through Flowering Locus $\mathrm{T}$ to promote flowering in Arabidopsis. Plant Physiol 2005, 139(2):770-778.

14. Amasino R: Seasonal and developmental timing of flowering. Plant J 2010, 61(6):1001-1013.

15. Helliwell CA, Wood CC, Robertson M, Peacock WJ, Dennis ES: The Arabidopsis FLC protein interacts directly in vivo with SOC1 and FT chromatin and is part of a high-molecular-weight protein complex. Plant J 2006, 46(2):183-192.

16. Blazquez MA, Green R, Nilsson O, Sussman MR, Weigel D: Gibberellins promote flowering of Arabidopsis by activating the LEAFY promoter. Plant Cell Online 1998, 10(5):791-800.

17. Brunner AM, Nilsson O: Revisiting tree maturation and floral initiation in the poplar functional genomics era. New Phytologist 2004, 164(1):43-51.

18. Talon M, Gmitter FG Jr: Citrus genomics. Int J Plant Genomics 2008, 2008:1-17.

19. Marques MC, Alonso-Cantabrana H, Forment J, Arribas R, Alamar S, Conejero V, Perez-Amador MA: A new set of ESTs and CDNA clones from full-length and normalized libraries for gene discovery and functional characterization in citrus. BMC Genomics 2009, 10(1):428.

20. Pe aL, Martín-Trillo M, Juárez J, Pina JA, Navarro L, Martínez-Zapater JM: Constitutive expression of Arabidopsis LEAFY or APETALA1 genes in citrus reduces their generation time. Nat Biotechnol 2001, 19(3):263-267.

21. Endo T, Shimada T, Fujii H, Kobayashi Y, Araki T, Omura M: Ectopic expression of an FT homolog from Citrus confers an early flowering phenotype on trifoliate orange (Poncirus trifoliata L. Raf.). Transgenic Res 2005, 14(5):703-712.

22. Zhang JZ, Li ZM, Mei L, Yao JL, Hu CG: PtFLC homolog from trifoliate orange (Poncirus trifoliata) is regulated by alternative splicing and experiences seasonal fluctuation in expression level. Planta 2009, 229(4):847-859.

23. Zhang JZ, Li ZM, Liu L, Mei L, Yao JL, Hu CG: Identification of early-flowerrelated ESTs in an early-flowering mutant of trifoliate orange (Poncirus trifoliata) by suppression subtractive hybridization and macroarray analysis. Tree Physiol 2008, 28(10):1449-1457.

24. Zhang JZ, Li ZM, Yao JL, Hu CG: Identification of flowering-related genes between early flowering trifoliate orange mutant and wild-type trifoliate orange (Poncirus trifoliata L. Raf.) by suppression subtraction hybridization (SSH) and macroarray. Gene 2009, 430(1-2):95-104. 
25. Li ZM, Zhang JZ, Mei L, Deng XX, Hu CG, Yao JL: PtSVP, an SVP homolog from trifoliate orange (Poncirus trifoliata L. Raf.), shows seasonal periodicity of meristem determination and affects flower development in transgenic Arabidopsis and tobacco plants. Plant Mol Biol 2010, 74:129-142.

26. Adams MD, Kerlavage AR, Fleischmann RD, Fuldner RA, Bult CJ, Lee NH, Kirkness EF, Weinstock KG, Gocayne JD, White O: Initial assessment of human gene diversity and expression patterns based upon 83 million nucleotides of cDNA sequence. Nature 1995, 377(6547 Suppl):371-374.

27. Velculescu VE, Zhang L, Vogelstein B, Kinzler KW: Serial analysis of gene expression. Science 1995, 270(5235):484-487

28. Meyers BC, Vu TH, Tej SS, Ghazal H, Matvienko M, Agrawal V, Ning J, Haudenschild CD: Analysis of the transcriptional complexity of Arabidopsis thaliana by massively parallel signature sequencing. Nat Biotechnol 2004, 22(8):1006-1011.

29. Brenner S, Johnson M, Bridgham J, Golda G, Lloyd DH, Johnson D, Luo S, McCurdy S, Foy M, Ewan M: Gene expression analysis by massively parallel signature sequencing (MPSS) on microbead arrays. Nat Biotechnol 2000, 18(6):630-634.

30. Meyers BC, Tej SS, Vu TH, Haudenschild CD, Agrawal V, Edberg SB, Ghazal H, Decola S: The use of MPSS for whole-genome transcriptional analysis in Arabidopsis. Genome Res 2004, 14(8):1641-1653.

31. Iandolino A, Nobuta K, Da Silva FG, Cook DR, Meyers BC: Comparative expression profiling in grape (Vitis vinifera) berries derived from frequency analysis of ESTs and MPSS signatures. BMC Plant Biol 2008, 8(1):53.

32. Guo M, Yang S, Rupe M, Hu B, Bickel DR, Arthur L, Smith O: Genome-wide allele-specific expression analysis using Massively Parallel Signature Sequencing (MPSS(tm)) Reveals cis-and trans-effects on gene expression in maize hybrid meristem tissue. Plant Mol Biol 2008, 66(5):551-563.

33. Meyers BC, Tej SS, Vu TH, Haudenschild CD, Agrawal V, Edberg SB, Ghazal H, Decola S: The use of MPSS for whole-genome transcriptional analysis in Arabidopsis. Genome Res 2004, 14(8):1641.

34. Kal AJ, Van Zonneveld AJ, Benes V, Van Den Berg M, Koerkamp MG, Albermann K, Strack N, Ruijter JM, Richter A, Dujon B: Dynamics of gene expression revealed by comparison of serial analysis of gene expression transcript profiles from yeast grown on two different carbon sources. Mol Biol Cell 1999, 10(6):1859-1872.

35. Yamada K, Lim J, Dale JM, Chen H, Shinn P, Palm CJ, Southwick AM, Wu HC, Kim C, Nguyen M: Empirical analysis of transcriptional activity in the Arabidopsis genome. Science 2003, 302(5646):842-846.

36. Umezawa T, Sakurai T, Totoki Y, Toyoda A, Seki M, Ishiwata A, Akiyama K, Kurotani A, Yoshida T, Mochida K: Sequencing and analysis of approximately 40000 soybean cDNA clones from a full-length-enriched cDNA library. DNA Res 2008, 15(6):333-346.

37. Alexandrov NN, Troukhan ME, Brover W, Tatarinova T, Flavell RB, Feldmann KA: Features of Arabidopsis genes and genome discovered using full-length cDNAs. Plant Mol Biol 2006, 60(1):69-85.

38. Ralph SG, Chun HJE, Cooper D, Kirkpatrick R, Kolosova N, Gunter L, Tuskan GA, Douglas CJ, Holt RA, Jones SJM: Analysis of 4,664 high-quality sequence-finished poplar full-length CDNA clones and their utility for the discovery of genes responding to insect feeding. BMC Genomics 2008, 9(1):57.

39. Jia J, Fu J, Zheng J, Zhou $X$, Huai J, Wang J, Wang M, Zhang $Y$, Chen $X$, Zhang J: Annotation and expression profile analysis of 2073 full-length CDNAs from stress-induced maize (Zea mays L.) seedlings. Plant J 2006 48(5):710-727.

40. Alexandrov NN, Brover W, Freidin S, Troukhan ME, Tatarinova TV, Zhang H, Swaller TJ, Lu YP, Bouck J, Flavell RB: Insights into corn genes derived from large-scale cDNA sequencing. Plant Mol Biol 2009, 69(1):179-194

41. Iseli C, Jongeneel CV, Bucher P: ESTScan: a program for detecting, evaluating, and reconstructing potential coding regions in EST sequences. International Conference on Intelligent Systems for Molecular Biology: 1999 1999, 138-148.

42. Matys V, Kel-Margoulis OV, Fricke E, Liebich I, Land S, Barre-Dirrie A, Reuter I, Chekmenev D, Krull M, Hornischer K: TRANSFAC (R) and its module TRANSCompel (R): transcriptional gene regulation in eukaryotes. Nucleic Acids Res 2006, 34(Database Issue):D108.
43. Guo AY, Chen X, Gao G, Zhang H, Zhu QH, Liu XC, Zhong YF, Gu X, He K, Luo J: PlantTFDB: a comprehensive plant transcription factor database. Nucleic Acids Res 2008, 36(Database issue):D966.

44. Haas BJ, Delcher AL, Mount SM, Wortman JR, Smith RK Jr, Hannick LI, Maiti R, Ronning CM, Rusch DB, Town CD: Improving the Arabidopsis genome annotation using maximal transcript alignment assemblies. Nucleic Acids Res 2003, 31(19):5654

45. He $Y$, Michaels SD, Amasino RM: Regulation of flowering time by histone acetylation in Arabidopsis. Science 2003, 302(5651):1751.

46. Pillitteri LJ, Lovatt CJ, Walling LL: Isolation and characterization of LEAFY and APETALA1 homologues from Citrus sinensis L. Osbeck'Washington'. J Am Soc Hort Sci 2004, 129:846-856.

47. Iandolino A, Nobuta K, Da Silva F, Cook D, Meyers B: Comparative expression profiling in grape (Vitis vinifera) berries derived from frequency analysis of ESTs and MPSS signatures. BMC Plant Biol 2008, 8(1):53.

48. Xu Q, Yu K, Zhu A, Ye J, Liu Q, Zhang J, Deng X: Comparative transcripts profiling reveals new insight into molecular processes regulating lycopene accumulation in a sweet orange (Citrus sinensis) red-flesh mutant. BMC Genomics 2009, 10(1):540.

49. Hoth S, Morgante M, Sanchez JP, Hanafey MK, Tingey SV, Chua NH: Genome-wide gene expression profiling in Arabidopsis thaliana reveals new targets of abscisic acid and largely impaired gene regulation in the abi1-1 mutant. J Cell Sci 2002, 115(24):4891-4900.

50. Fontanillas P, Landry CR, Wittkopp PJ, Russ C, Gruber JD, Nusbaum C, Hartl DL: Key considerations for measuring allelic expression on a genomic scale using high-throughput sequencing. Mol Ecol 2010, 19(s1):212-227.

51. Jongeneel CV, Iseli C, Stevenson BJ, Riggins GJ, Lal A, Mackay A, Harris RA, O'Hare MJ, Neville AM, Simpson AJG: Comprehensive sampling of gene expression in human cell lines with massively parallel signature sequencing. Proc Natl Acad Sci USA 2003, 100(8):4702-4705.

52. Jongeneel CV, Delorenzi M, Iseli C, Zhou D, Haudenschild CD, Khrebtukova I, Kuznetsov D, Stevenson BJ, Strausberg RL, Simpson AJG: An atlas of human gene expression from massively parallel signature sequencing (MPSS). Genome Res 2005, 15(7):1007-1014.

53. Meyers BC, Lee DK, Vu TH, Tej SS, Edberg SB, Matvienko M, Tindell LD: Arabidopsis MPSS. An online resource for quantitative expression analysis. Plant Physiol 2004, 135(2):801-813.

54. Hedgecock D, Lin JZ, DeCola S, Haudenschild CD, Meyer E, Manahan DT, Bowen B: Transcriptomic analysis of growth heterosis in larval Pacific oysters (Crassostrea gigas). Proc Natl Acad Sci 2007, 104(7):2313-2318

55. Koshita Y, Takahara T, Ogata T, Goto A: Involvement of endogenous plant hormones (IAA, ABA, GAs) in leaves and flower bud formation of satsuma mandarin (Citrus unshiu Marc.). Scientia Horticulturae 1999, 79(3-4):185-194.

56. McArtney SJ: Exogenous gibberellin affects biennial bearing and the fruit shape of 'Braeburn' apple. New Zealand J Crop Hort Sci 1994, 22(3):343-346.

57. Inglese P, Chessa I, La Mantia T, Nieddu G: Evolution of endogenous gibberellins at different stages of flowering in relation to return bloom of cactus pear (Opuntia ficus-indica L. Miller). Scientia Horticulturae 1998, 73(1):45-51.

58. Anderson JL, Seeley SD: Bloom delay in deciduous fruits. Hort Rev 1993, 15:97-144.

59. Lee J, Lee I: Regulation and function of SOC1, a flowering pathway integrator. J Exp Bot 2010, 61(9):2247-2254.

60. Tan FC, Swain SM: Functional characterization of AP3, SOC1 and WUS homologues from citrus (Citrus sinensis). Physiologia Plantarum 2007, 131(3):481-495.

61. Cervera M, Navarro L, Pe aL: Gene stacking in 1-year-cycling APETALA1 citrus plants for a rapid evaluation of transgenic traits in reproductive tissues. J Biotechnol 2009, 140(3-4):278-282.

62. Valverde F, Mouradov A, Soppe W, Ravenscroft D, Samach A, Coupland G: Photoreceptor regulation of CONSTANS protein in photoperiodic flowering. Science 2004, 303(5660):1003.

63. Reeves PA, He Y, Schmitz RJ, Amasino RM, Panella LW, Richards CM: Evolutionary conservation of the FLOWERING LOCUS C-mediated 
vernalization response: evidence from the sugar beet (Beta vulgaris). Genetics 2007, 176(1):295-307.

64. Chandler J, Wilson A, Dean C: Arabidopsis mutants showing an altered response to vernalization. Plant J 1996, 10(4):637-644.

65. Sheldon CC, Rouse DT, Finnegan EJ, Peacock WJ, Dennis ES: The molecular basis of vernalization: the central role of FLOWERING LOCUS C (FLC). Proc Natl Acad Sci USA 2000, 97(7):3753-3758.

66. Erdner DL, Anderson DM: Global transcriptional profiling of the toxic dinoflagellate Alexandrium fundyense using Massively Parallel Signature Sequencing. BMC Genomics 2006, 7(1):88.

67. Saha S, Sparks AB, Rago C, Akmaev V, Wang CJ, Vogelstein B, Kinzler KW Velculescu VE: Using the transcriptome to annotate the genome. Nat Biotechnol 2002, 20(5):508-512.

68. Boss PK, Bastow RM, Mylne JS, Dean C: Multiple pathways in the decision to flower: enabling, promoting, and resetting. Plant Cell Online 2004, 16(suppl_1):S18.

69. Higgins JA, Bailey PC, Laurie DA, Hazen SP: Comparative genomics of flowering time pathways using Brachypodium distachyon as a model for the temperate grasses. PLoS One 2010, 5(4):e10065.

doi:10.1186/1471-2164-12-63

Cite this article as: Zhang et al: Transcriptome profile analysis of flowering molecular processes of early flowering trifoliate orange mutant and the wild-type [Poncirus trifoliata (L.) Raf.] by massively parallel signature sequencing. BMC Genomics 2011 12:63.

\section{Submit your next manuscript to BioMed Central and take full advantage of:}

- Convenient online submission

- Thorough peer review

- No space constraints or color figure charges

- Immediate publication on acceptance

- Inclusion in PubMed, CAS, Scopus and Google Scholar

- Research which is freely available for redistribution

Submit your manuscript at www.biomedcentral.com/submit 